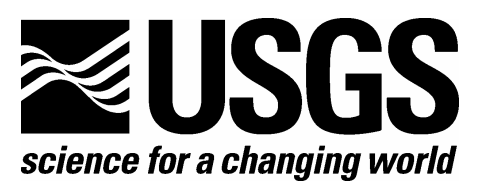

\title{
Genetic Analyses of Captive 'Alalā (Corvus hawaiiensis) Using AFLP Analyses
}

By Susan I. Jarvi and Kiara R. Bianchi

Open-File Report 2006-1349

U.S. Department of the Interior

U.S. Geological Survey 


\section{U.S. Department of the Interior \\ DIRK KEMPTHORENE, Secretary \\ U.S. Geological Survey \\ Mark D. Myers, Director}

U.S. Geological Survey, Reston, Virginia 2006

For product and ordering information:

World Wide Web: http://www.usgs.gov/pubprod

Telephone: 1-888-ASK-USGS

For more information on the USGS - the Federal source for science about the Earth, its natural and living resources, natural hazards, and the environment:

World Wide Web: http://www.usgs.gov

Telephone: 1-888-ASK-USGS

Suggested citation:

Jarvi, S.I., and Bianchi, K.R., 2006, Genetic Analyses of Captive 'Alalā (Corvus

hawaiiensis) using AFLP Analyses: U.S. Geological Survey Open-File

Report 2006-1349, 37 p. [http://pubs.usgs.gov/of/2006/1349/].

Any use of trade, product or firm names is for descriptive purposes only and does not imply endorsement by the U.S. Government.

Although this report is in the public domain, permission must be secured from the individual copyright owners to reproduce any copyrighted material contained within this report. 


\section{Contents}

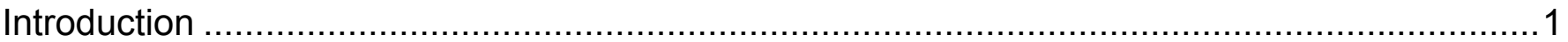

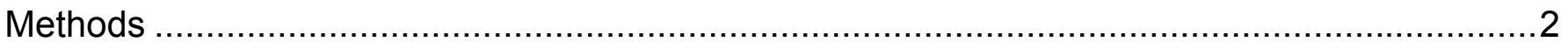

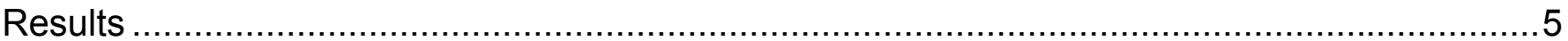

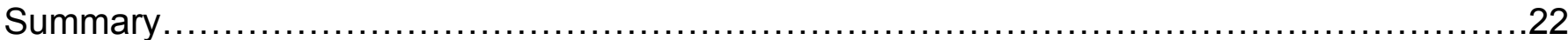

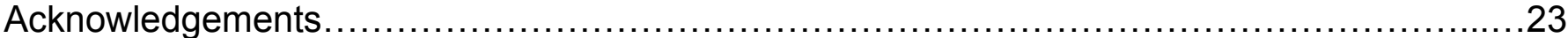

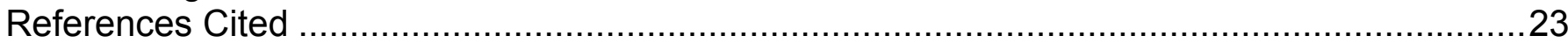

\section{Figures}

1. Primer screen with 6 sets of primers (M55, M57, M59, M60, M61, M62). All bands included (top) Primer screen 6 sets, polymorphic bands only (bottom). ......................... 7

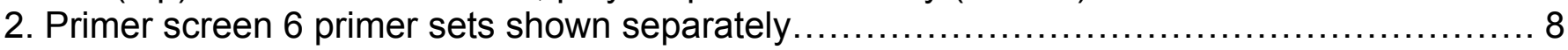

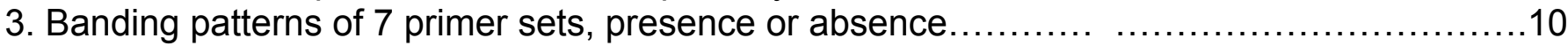

4. Phylogenetic relationships using 7 primer sets, all bands, Dice/UPGMA, no 'Umi.............18

5. Phylogenetic relationships using 7 primer sets, polymorphic bands only Dice/UPGMA ....... 19

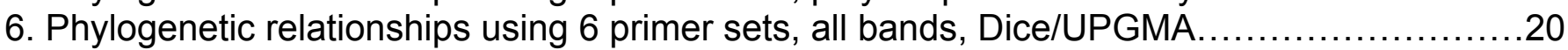

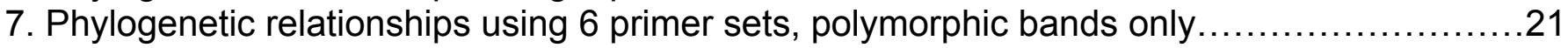

\section{Tables}

1. Total list of 49 extracted samples. The 41 used in final analyses are indicated.......................

2. Final analyses primer sets, total number of bands scored and frequency of polymorphic bands.

3. Summary of the similarity matrix depicting relationships among 5 individuals used in primer screens.

4. Binary table summary of pattern designation based on presence (1) or absence (0) of

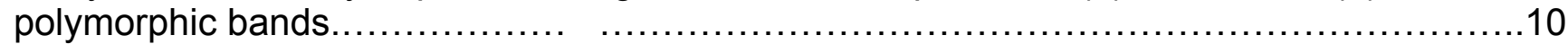

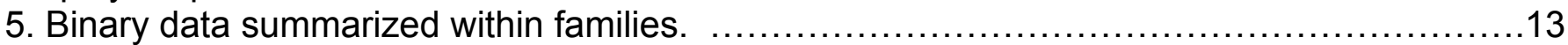

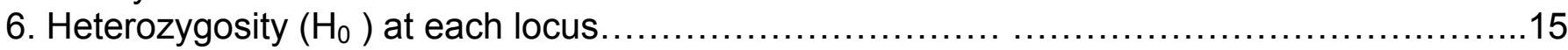

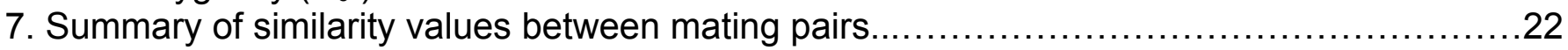

\section{Appendix}

Details of AFLP procedures.

AFLP Timeline. 


\title{
Genetic Analyses of Captive 'Alalā (Corvus hawaiiensis) Using AFLP Analysis
}

\author{
By Susan I. Jarvi ${ }^{1}$ and Kiara Bianchi ${ }^{1}$
}

\section{Introduction}

Population level studies of genetic diversity can provide information about population structure, individual genetic distinctiveness and former population size. They are especially important for rare and threatened species like the 'Alalā, where they can be used to assess extinction risks and evolutionary potential. In an ideal situation multiple methods should be used to detect variation, and these methods should be comparable across studies. In this report, we discuss AFLP (Amplified Fragment Length Polymorphism) as a genetic approach for detecting variation in the 'Alalā, describe our findings, and discuss these in relation to mtDNA and microsatellite data reported elsewhere in this same population (Fleisher, 2003).

AFLP is a technique for DNA fingerprinting that has wide applications (Vos et al., 1995). Because little or no prior knowledge of the particular species is required to carry out this method of analysis, AFLP can be used universally across varied taxonomic groups. Within individuals, estimates of diversity or heterozygosity across genomes may be complex because levels of diversity differ between and among genes (Sachidanandam et al., 2001; Bensch and Akesson, 2005). One of the more traditional methods of estimating diversity employs the use of codominant markers such as microsatellites. Codominant markers detect each allele at a locus independently. Hence, one can readily distinguish heterozygotes from homozygotes, directly assess allele frequencies and calculate other population level statistics. Dominant markers (e.g., AFLP) are scored as either present or absent (null) so heterozygotes cannot be directly distinguished from homozygotes. However, the presence or absence data can be converted to expected heterozygosity estimates which are comparable to those determined by codominant markers. High allelic diversity and heterozygosity inherent in microsatellites make them excellent tools for studies of wild populations and they have been used extensively. One limitation to the use of microsatellites is that heterozygosity estimates are affected by the mutation rate at microsatellite loci, thus introducing a bias. Also, the number of loci that can be studied is frequently limited to fewer than 10. This theoretically represents a maximum of one marker for each of 10 chromosomes.

Dominant markers like AFLP allow a larger fraction of the genome to be screened. Large numbers of loci can be screened by AFLP to resolve very small individual differences that can be used for identification of individuals, estimates of pairwise relatedness and, in some cases, for parentage analyses. Since AFLP is a dominant marker (can not distinguish between $+/+$ homozygote versus $+/$ - heterozygote), it has limitations for parentage analyses. Only when both parents are homozygous for the absence of alleles (-/-) and offspring show a presence (+/+ or $+/-)$ can the parents be excluded. In this case, microsatellites become preferable as they have the potential to

\footnotetext{
${ }^{1}$ Biology Department, University of Hawaii at Hilo, 200 West Kawili Street, Hilo, HI 96720
} 
exclude individual parents when the other parent is unknown. Another limitation of AFLP is that the loci are generally less polymorphic (only two alleles/locus) than microsatellite loci (often $>10$ alleles/locus). While generally fewer than 10 highly polymorphic microsatellite loci are enough to exclude and assign parentage, it might require up to 100 or more AFLP loci (reviewed in Bensch and Akesson, 2005). While there are pros and cons to different methodologies, the total number of loci evaluated by AFLP generally offsets the limitations imposed due to the dominant nature of this approach and end results between methods are generally comparable.

Overall objectives of this study were to evaluate the level of genetic diversity in the captive population of 'Alalā, to compare genetic data with currently available pedigree information, and to determine the extent of relatedness of mating pairs and among founding individuals.

\section{Methods}

DNA samples and extractions: Tissue samples were provided by the Zoological Society of San Diego, the Maui Bird Conservation Center, and the Keauhou Bird Conservation Center. Tissues were stored in screw-capped tubes containing $500 \mu \mathrm{L}$ of DNA lysis buffer $(0.1 \mathrm{M}$ Tris- $\mathrm{HCl}$, $\mathrm{pH}$ 8.0, 0.1M sodium EDTA, 2\% SDS) until extracted. Extractions were completed between 2001 and 2003 by either standard organic methods using phenol/chloroform or by column using the Qiagen DNeasy Kit. Those that were initially extracted by organic methods were subsequently reextracted using the column method for consistency. Several of the samples were also concentrated using "Zymo" columns to increase DNA concentration. All samples originated from blood or muscle except two that were from a yellowish, granular substance provided as necropsy samples. A total of 49 samples were extracted (Table 1).

Table 1. Blood samples that were extracted and used in final analyses (X).

\begin{tabular}{|c|c|c|c|c|c|}
\hline Sample & Studbook \# & DNA Log \# & Name & Extraction Date & Final Analysis \\
\hline 1 & 7 & 8353 & 'Umi & 18-Jun-02 & $\mathrm{X}$ \\
\hline 2 & 13 & $9614 / 9760$ & Lu’ukia & & $X$ \\
\hline 3 & 17 & $9613 / 9759$ & Mana & & \\
\hline 4 & 25 & 6933 & Kolohe & 21-Nov-01 & $X$ \\
\hline 5 & 26 & 8352 & Keli`i & 18-Jun-02 & $\mathrm{X}$ \\
\hline 6 & 27 & 6943 & Kalani & 21-Nov-01 & $X$ \\
\hline 7 & 28 & 6934 & Keawe & 21-Nov-01 & $\mathrm{X}$ \\
\hline 8 & 30 & 6935 & Wa'alani & 21-Nov-01 & $\mathrm{X}$ \\
\hline 9 & 31 & 6936 & Ho`okū & 21-Nov-01 & $\mathrm{X}$ \\
\hline 10 & 32 & 6937 & Hō`ikeikapo & 21-Nov-01 & $\mathrm{X}$ \\
\hline 11 & 33 & 6938 & Kīnohi & 21-Nov-01 & $X$ \\
\hline 12 & 35 & 6939 & Lanakila & 21-Nov-01 & $X$ \\
\hline \multirow[t]{2}{*}{13} & 36 & 7155 & Hiwahiwa & 11-Feb-02 & $X$ \\
\hline & 36 & $1377-9$ & Hiwahiwa & 05-Mar-97 & \\
\hline \multirow[t]{2}{*}{14} & 37 & 7154 & Hoapili & $11-F e b-02$ & $\mathrm{X}$ \\
\hline & 37 & $1374-6$ & Hoapili & 05-Mar-97 & \\
\hline
\end{tabular}




\begin{tabular}{|c|c|c|c|c|c|}
\hline \multirow[t]{2}{*}{15} & 38 & 6944 & Lōkāhi & 21-Nov-01 & $\mathrm{X}$ \\
\hline & 38 & 7171 & Lōkāhi & 11-Feb-02 & \\
\hline \multirow[t]{2}{*}{16} & 39 & 7156 & Mālama & 11-Feb-02 & $y$ \\
\hline & 39 & $1380-2$ & Mālama & 05-Mar-97 & \\
\hline 17 & 40 & 7172 & Hulali & 11-Feb-02 & \\
\hline \multirow[t]{4}{*}{18} & 41 & 6945 & Kēhau & 21-Nov-01 & \\
\hline & 41 & 7157 & Kēhau & 11-Feb-02 & \\
\hline & 41 & 9940 & Kēhau & 25-Mar-03 & \\
\hline & 41 & $1383-5$ & Kēhau & 05-Mar-97 & \\
\hline 19 & 54 & 6946 & Pōmaika`i & 21-Nov-01 & $X$ \\
\hline 20 & 56 & 6947 & Leinani & 21-Nov-01 & v \\
\hline 21 & 57 & 6948 & 'Ula & 21-Nov-01 & \\
\hline \multirow[t]{3}{*}{22} & 58 & 7152 & Noe & 11-Feb-02 & \\
\hline & 58 & $1398-1407$ & Noe & 12-Apr-97 & \\
\hline & 58 & $4116-18$ & Noe & 07-Mar-98 & \\
\hline \multirow[t]{3}{*}{23} & 61 & 7160 & Hilu & 11-Feb-02 & v \\
\hline & 61 & $1408-1417$ & Hilu & 12-Apr-97 & \\
\hline & 61 & $4119-21$ & Hilu & 07-Mar-98 & \\
\hline 24 & 66 & 6940 & Nīele & 21-Nov-01 & $X$ \\
\hline \multirow[t]{2}{*}{25} & 67 & 6942 & 'Oli & 21-Nov-01 & $y$ \\
\hline & 67 & 9939 & 'Oli & 25-Mar-03 & \\
\hline \multirow[t]{3}{*}{26} & 68 & 7158 & Uila & 11-Feb-02 & \\
\hline & 68 & $4080-2$ & Uila & 07-Mar-98 & \\
\hline & 68 & $1386-8$ & Uila & 05-Mar-97 & \\
\hline \multirow[t]{3}{*}{27} & 69 & 7170 & `Ele`ele & 11-Feb-02 & $X$ \\
\hline & 69 & $1392-5$ & `Ele`ele & 05-Mar-97 & \\
\hline & 69 & $4086-8$ & `Ele`ele & 07-Mar-98 & \\
\hline \multirow[t]{2}{*}{28} & 70 & 7164 & Makuni & 11-Feb-02 & \\
\hline & 70 & $4083-5$ & Makuni & 07-Mar-98 & \\
\hline \multirow[t]{3}{*}{29} & 71 & 7159 & Konakū & 11-Feb-02 & \\
\hline & 71 & $1395-7$ & Konakū & 05-Mar-97 & \\
\hline & 71 & 4089-91 & Konakū & 07-Mar-98 & \\
\hline 30 & 73 & 6949 & Kekoa & 21-Nov-01 & $X$ \\
\hline \multirow[t]{3}{*}{31} & 74 & 7153 & Hiapo & 11-Feb-02 & $X$ \\
\hline & 74 & 9938 & Hiapo & 25-Mar-03 & \\
\hline & 74 & $4128-33$ & Hiapo & 07-Mar-98 & \\
\hline 32 & 75 & 6950 & Laha & 21-Nov-01 & 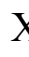 \\
\hline \multirow[t]{2}{*}{33} & 76 & 7163 & Makoa & 11-Feb-02 & $\pi$ \\
\hline & 76 & $4074-9$ & Makoa & 07-Mar-98 & \\
\hline \multirow[t]{2}{*}{34} & 77 & 7169 & Puanani & 11-Feb-02 & \\
\hline & 77 & $4125-27$ & Puanani & 07-Mar-98 & \\
\hline
\end{tabular}




\begin{tabular}{|c|c|c|c|c|c|}
\hline \multirow[t]{3}{*}{35} & 78 & 7168 & Ao & 11-Feb-02 & \multirow[t]{3}{*}{$\mathrm{X}$} \\
\hline & 78 & 9941 & Ao & 25-Mar-03 & \\
\hline & 78 & $4113-15$ & Ao & 07-Mar-98 & \\
\hline \multirow[t]{2}{*}{36} & 79 & 7167 & Kahuli & 11-Feb-02 & \multirow[t]{2}{*}{$\mathrm{X}$} \\
\hline & 79 & $4122-24$ & Kahuli & 07-Mar-98 & \\
\hline \multirow[t]{2}{*}{37} & 80 & 7166 & Hulu & 11-Feb-02 & \multirow[t]{4}{*}{$\mathrm{X}$} \\
\hline & 80 & $4107-9$ & Hulu & 07-Mar-98 & \\
\hline \multirow[t]{2}{*}{38} & 81 & 7165 & Lehua & 11-Feb-02 & \\
\hline & 81 & 4104-6 & Lehua & 07-Mar-98 & \\
\hline \multirow[t]{3}{*}{39} & 82 & 6941 & Kauila & 21-Nov-01 & \multirow[t]{3}{*}{$\mathrm{X}$} \\
\hline & 82 & 7151 & Kauila & 11-Feb-02 & \\
\hline & 82 & $4110-12$ & Kauila & 07-Mar-98 & \\
\hline 40 & 86 & 6951 & Kilakila & 21-Nov-01 & $\mathrm{X}$ \\
\hline 41 & 87 & 6952 & Hōkūlele & 21-Nov-01 & $\mathrm{X}$ \\
\hline 42 & 88 & 6953 & 'Oni`oni & 21-Nov-01 & $\mathrm{X}$ \\
\hline 43 & 89 & 6954 & Pīkoi & 21-Nov-01 & $X$ \\
\hline 44 & 90 & 6955 & Akamai & 21-Nov-01 & $\mathrm{X}$ \\
\hline 45 & 91 & 6956 & Mai’aokea & 21-Nov-01 & $\mathrm{X}$ \\
\hline 46 & 92 & 6957 & Pīkaka & 21-Nov-01 & $\mathrm{X}$ \\
\hline \multirow[t]{3}{*}{47} & & 7161 & 12474(\#4) & 11-Feb-02 & \\
\hline & & $4053-5$ & 212474 & 07-Mar-98 & \\
\hline & & $5428-30$ & 12474 & 29-Sep-98 & \\
\hline \multirow[t]{2}{*}{48} & & 7162 & 12475(\#1) & 11-Feb-02 & \\
\hline & & $4056-8$ & $412475-1$ & 07-Mar-98 & \\
\hline 49 & & 1389-91 & NB & 05-Mar-97 & \\
\hline
\end{tabular}

AFLP: AFLP consists of four basic steps: (1) restriction enzyme digest of genomic DNA, (2) attachment of short DNA segments (adapters) to the ends of all digested fragments, (3) amplification of fragments using selective primers, of which one is labeled for later visualization, and (4) separation and visualization of amplified fragments by gel electrophoresis. While the protocol for AFLP appears quite straightforward, optimization is required to obtain high quality, consistent and replicable fingerprint patterns. Of particular importance is consistency in the quality and quantity of starting genomic DNA and the success of the ligation (attachment) reaction, which provides the priming sites for selective amplification. Steps (1), (2), and (3) (above) were completed at the University of Hawaii, Hilo, and step 4 (separation and visualization of products) was completed on an ABI genotyping system at the Biotech facility at the University of Hawaii, Manoa. For samples used in this study, genomic DNA was digested with restriction enzymes EcoR1 and Mse 1 which cleave at 6 base pair sites (Step 1). Adapters were ligated to the ends of all fragments (Step 2). Selective amplification was then performed to amplify subsets of this genomic "soup" depending on the 2 or 3 base pair sequence at the end of each primer (Step 3, Appendix A). For each set of primers, only one was labeled with a fluorescent dye (either TET, HEX or FAM) which fluoresces at distinct wavelengths. PCR products from these reactions, in addition to a 
known marker labeled with another dye (ROX) were run in polyacrylamide gels and bands were separated, identified by size and computerized (Step 4). We then evaluated the data in these files for repeatability and overall consistency. Criteria for selecting bands and individuals to include in final analyses were based on repeatability and consistency and are provided in stepwise fashion in Appendix A. We initially identified the bands or band classes to include in the analysis for each primer set and then decided (Step 2 in Appendix) whether to keep or remove individuals based on repeatability and consistency of runs within individual primer sets. We next determined which bands to use in the final analysis (Step 3). Finally, we compiled data over multiple primer sets for the final analysis (Step 4). This step is integral with Step 2. All AFLP data analyses were completed using the program Bionumerics (Applied Maths), with the exception of heterozygosity estimates which were completed using the program Hickory (v.1.0.3).

Primer screens. Four individuals ('Ele'ele, Kauila, Noe, Hiapo), were screened initially in 2001 in triplicate with $6 \mathrm{Eco} / \mathrm{Taq}$ primer sets (A, B, C, D, E, F). These samples were all extracted using organic methods. In 2002, we assayed 42 samples on 3 primer sets (M3, M5, M9), trying a new enzyme set, Eco/Mse. Low, but detectable, levels of polymorphism were observed. We determined that all samples required re-extraction by column DNA isolation. We subsequently screened 36 new primers on samples from Keawe, Wa'alani, 'Oli, Pōmaika'i, and Laha which were digested with Eco/Mse. Of these 45 primer sets, 7 were selected for further population analyses.

\section{Results}

While samples from 49 individuals were available, we report results from 41 individuals (as indicated in Table 1). The primer sets used in the final analyses, along with the size ranges of fragments and polymorphic bands are listed in Table 2 .

Table 2. Total number of bands scored and frequency of polymorphic bands in the final analyses of individual primer sets.

\begin{tabular}{ccccc}
\hline $\begin{array}{c}\text { Eco/Mse primer } \\
\text { set }\end{array}$ & $\begin{array}{c}\text { Total \# bands } \\
\text { scored }\end{array}$ & $\begin{array}{c}\text { Size Range } \\
(\mathrm{bp})\end{array}$ & $\begin{array}{c}\text { Total \# polymorphic } \\
\text { bands }\end{array}$ & $\begin{array}{c}\text { Frequency of } \\
\text { polymorphic bands }\end{array}$ \\
\hline M3 & 35 & $63-203$ & 1 & 0.029 \\
M5 & 63 & $59-256$ & 3 & 0.048 \\
M55 & 38 & $67-223$ & 2 & 0.053 \\
M57 & 35 & $55-250$ & 5 & 0.143 \\
M60 & 19 & $51-313$ & 6 & 0.316 \\
M61 & 50 & $54-192$ & 3 & 0.06 \\
M62 & 15 & $61-323$ & 5 & 0.333 \\
Total & 255 & & &
\end{tabular}


Four individuals that were evaluated for the initial primer screen in 2001 with 4 of the 6 primer sets (ABCD) were selected based on quantity and quality of available DNA and familial information provided by the U. S. Fish and Wildlife Service in the 2001 pedigree flow chart and studbook. According to the pedigree, Noe is a descendant of the Keâlia pair, Ele'ele and Kauila are descendants of the founders 'Umi and Lu' ukia, and Hiapo is an offspring of 'Ula (Kalāhiki pair) and Kalani ('Umi and Lu' ukia). While these four primer sets clearly differentiated these four individuals with diversity ranging from $82 \%-91 \%$ (data not shown), we had concerns with repeatability and reliability in this trial. Also of concern was the fact that the Taq enzyme we used is methylation sensitive, and could possible influence the results. These data were removed from further analyses.

The second screen was completed with three primer sets M3, M5 and M9 on 42 samples using the Eco/Mse enzyme digest. We detected low levels of polymorphism. However, we decided to re-extract some of the samples to provide uniformity of methods of all samples. The information from this screening was useful in establishing the overall diversity detected by these primers, and two of them (M3, M5) were selected for further analyses (described below). We did not use this scoring data in the final analyses, however, due to lack of reproducibility.

In the screening done with $36 \mathrm{Eco} / \mathrm{Mse}$ primer sets, initial evaluation of the banding patterns among replicates revealed that several were unscorable (faint or unclear bands) or too difficult to score reliably (bands present but do not distinct peaks i.e., have a "shoulder"), and we selected 6 sets to score. The resulting relationships among 5 individuals with these 6 selected primer sets (M55, M57, M59, M60, M61, M62) are presented in Figure 1. Results from these sets of primers are illustrated in Figure 2. 
Figure 1. Five individuals were selected for screening 36 sets of primers. The phylogenetic relationships among these five individuals based on six sets of primers are shown using all bands (upper, including monomorphic and polymorphic) and with only polymorphic (lower) with presence (shaded) or absence (non-shaded) of bands as indicated. Phylogenetic relationships were estimated by Dice methods and depicted by UPGMA (Unweighted Pair Group Method with Arithrimetic Mean). Primer/band class key (lower) is available upon request.

\section{Phylogenetic Relationships Based on All Bands for Primers M55+M57+M59+M60+M61+M62}
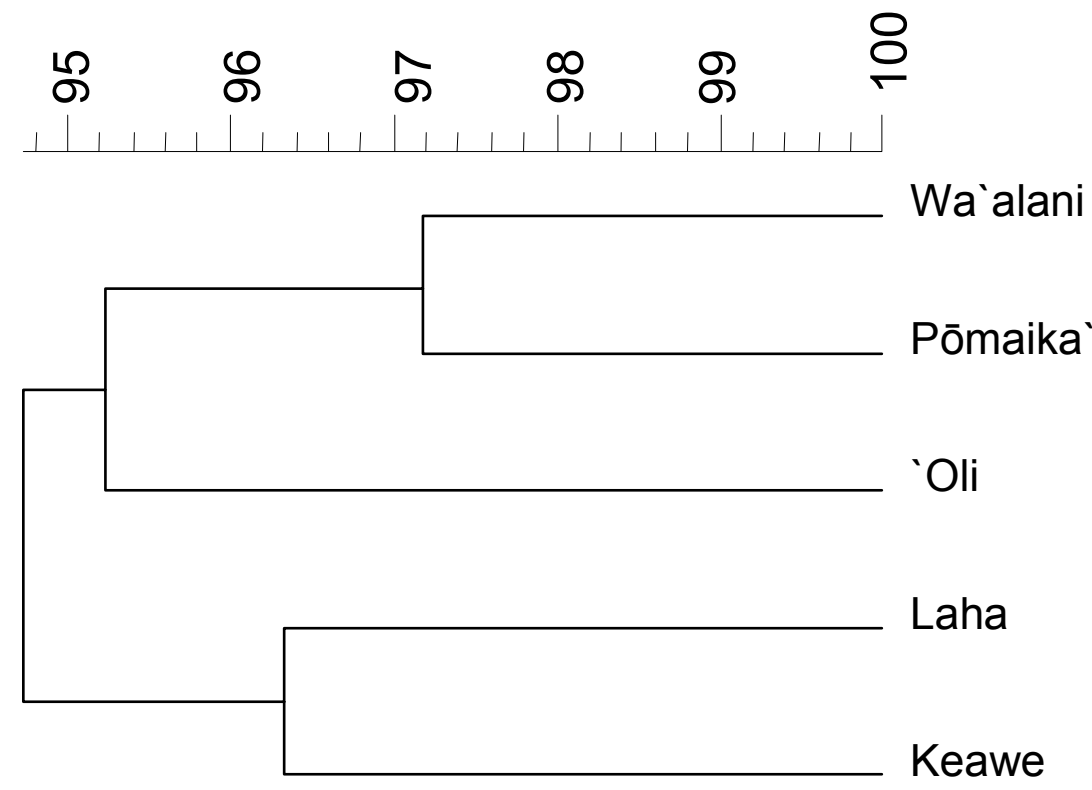

Phylogenetic Relationships Based on Polymorphic Bands for Primers $\mathrm{M} 55+\mathrm{M} 57+\mathrm{M} 59+\mathrm{M} 60+\mathrm{M} 61+\mathrm{M} 62$

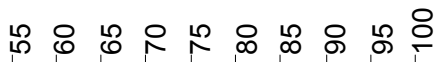

\section{Band Classes}

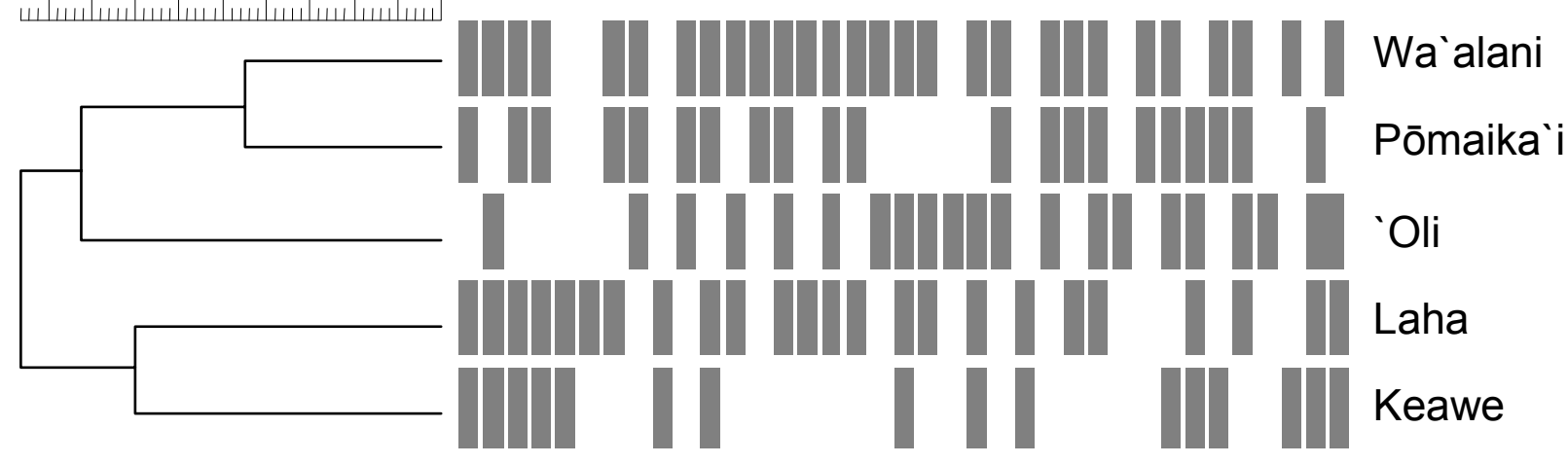


Figure 2. Phylogenetic relationships of 5 individuals involved in primer screens by individual primer sets. Primer sets are: M57, M59, M60, M61, M62, and M55. Distance estimates (Dice, Bionumerics) were used to depict phylogenetic relationships among these individuals in a UPGMA tree.

\section{Primer M57}

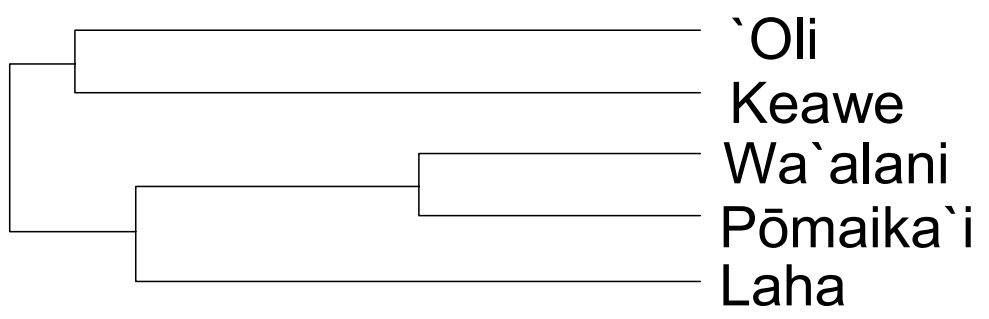

\section{Primer M59}

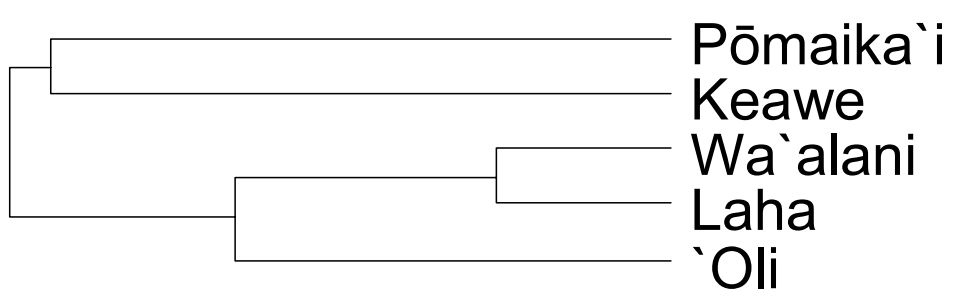

\section{Primer M60}

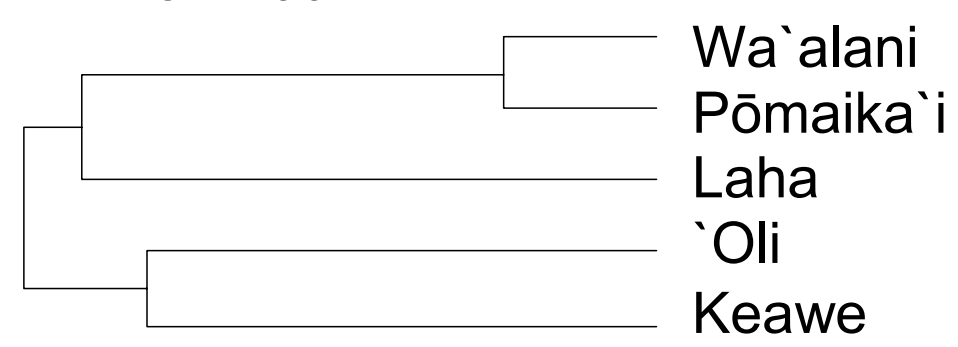

\section{Primer M61}

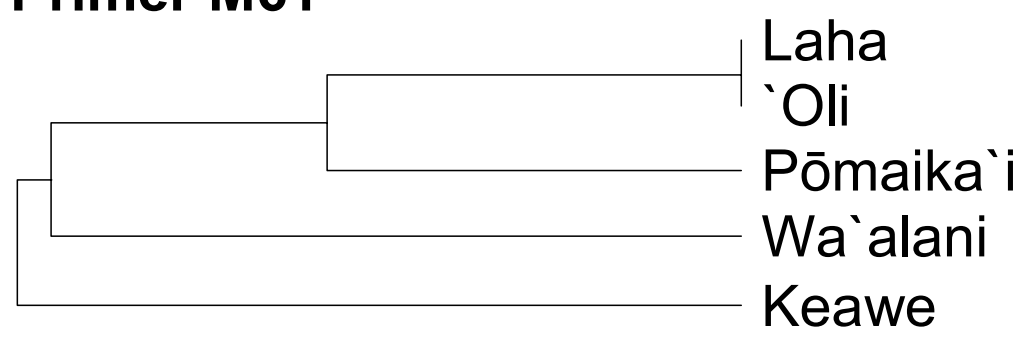

Primer M62
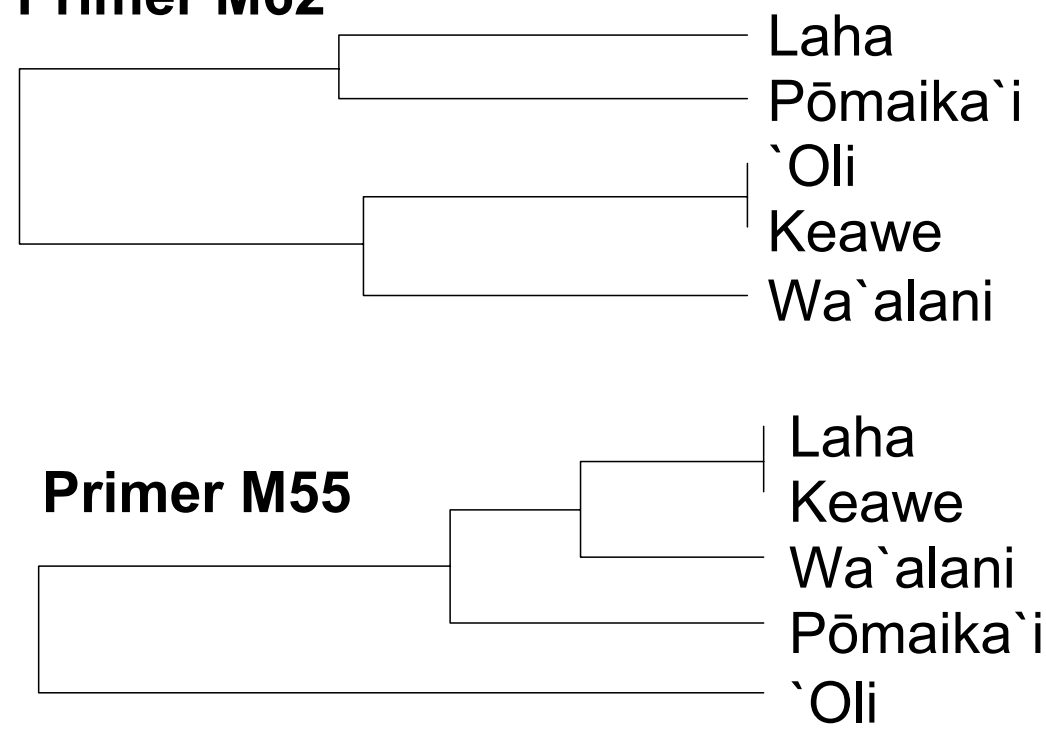
Individuals selected for inclusion were 'Oli from the Hookena pair, Pōmaika' $i$ from the Kalāhiki pair, Keawe from 'Umi and Lu'ukia, founder Wa`alani and Laha from 'Ula and Kalani. Of the total number of bands scored, 37 were polymorphic bands. A summary of the similarity matrix depicting relationships is shown in Table 3. Based on these similarity values, Pōmaika'i (McCandless) appears most similar to Wa'alani (Hu'alalāi descendent) and the least similar pair is Keawe ('Umi and Lu ukia offspring, Hu'alalāi) and 'Oli (Hookena pair, McCandless). Five of these primer pairs were selected for inclusion in the final analyses (M55, M57, M60, M61, M62). The similarity matrix depicting the relationships among these 5 individuals is shown in Table 3 .

Table 3. Similarity matrix depicting relationships among 5 individuals used in primer screen (M55, M57, M59, M60, M61, M62). Numbers shown are based on all bands / polymorphic bands only. The highest (most similar) and lowest (least similar) values are underlined.

\begin{tabular}{cccccc}
\hline & Wa`alani & Pōmaika`i & 'Oli & Laha & Keawe \\
\hline Wa`alani & $100 / 100$ & & & & \\
Pōmaika`i & $\underline{97.19 / 77.55}$ & $100 / 100$ & & & \\
'Oli & $97.19 / 65.31$ & $94.79 / 52.38$ & $100 / 100$ & & \\
Laha & $95.94 / 69.23$ & $95.09 / 57.78$ & $94.57 / 53.33$ & $100 / 100$ & \\
Keawe & $94.3 / 50.00$ & $94.46 / 43.24$ & $\underline{93.93 / 37.84}$ & $96.33 / 65$. & $100 / 100$ \\
\hline
\end{tabular}

A total of seven primer sets were used for further analyses (M3, M5, M55, M57, M60, M61, M62). Of the 49 total samples available, 41 were included in the final analyses (indicated in Table 1). Bands produced by these 7 sets of primers are scored as either present (1) or absent (0). We evaluated a total of 255 loci for polymorphism and out of these $9.8 \%$ were polymorphic (25 band classes using all 7 sets of primers, but only 20 band classes if primer set M57 is excluded see Table 2).

A total of 19 distinct banding patterns were distinguished among these 41 individuals. The polymorphic bands distinguishing these genetic patterns are summarized as a binary table (Table 4) and as presence or absence in Figure 3. Individuals of identical pattern are color-coded in Table 4, with 12 of the 41 individuals evaluated showing unique patterns. Of note is that founding individuals Wa'alani, 'Umi and Lu'ukia each appear distinct from one another.

Pattern 1 is the predominant pattern and includes individuals originating from several families and lineages (Table 5). Many of the remaining individuals are distinct from pattern one at only one locus and fall into Patterns 2, 3, 4, 5, 14 and 15. Individuals in Patterns 7, 9, 10, 11, 12, and 13 are distinct from the predominant pattern at two loci, Patterns 6, 8, 16 and 17 by three loci, Pattern 18 (Lu'ukia) at 5 loci and Pattern 19 ('Ele'ele) at 12 loci. 
Figure 3. Polymorphic banding patterns detected in 40 'Alala. Presence of bands are indicated by box shading, and absence by none. Band classes (A-Y) are defined in the key*. Studbook number follows each name.

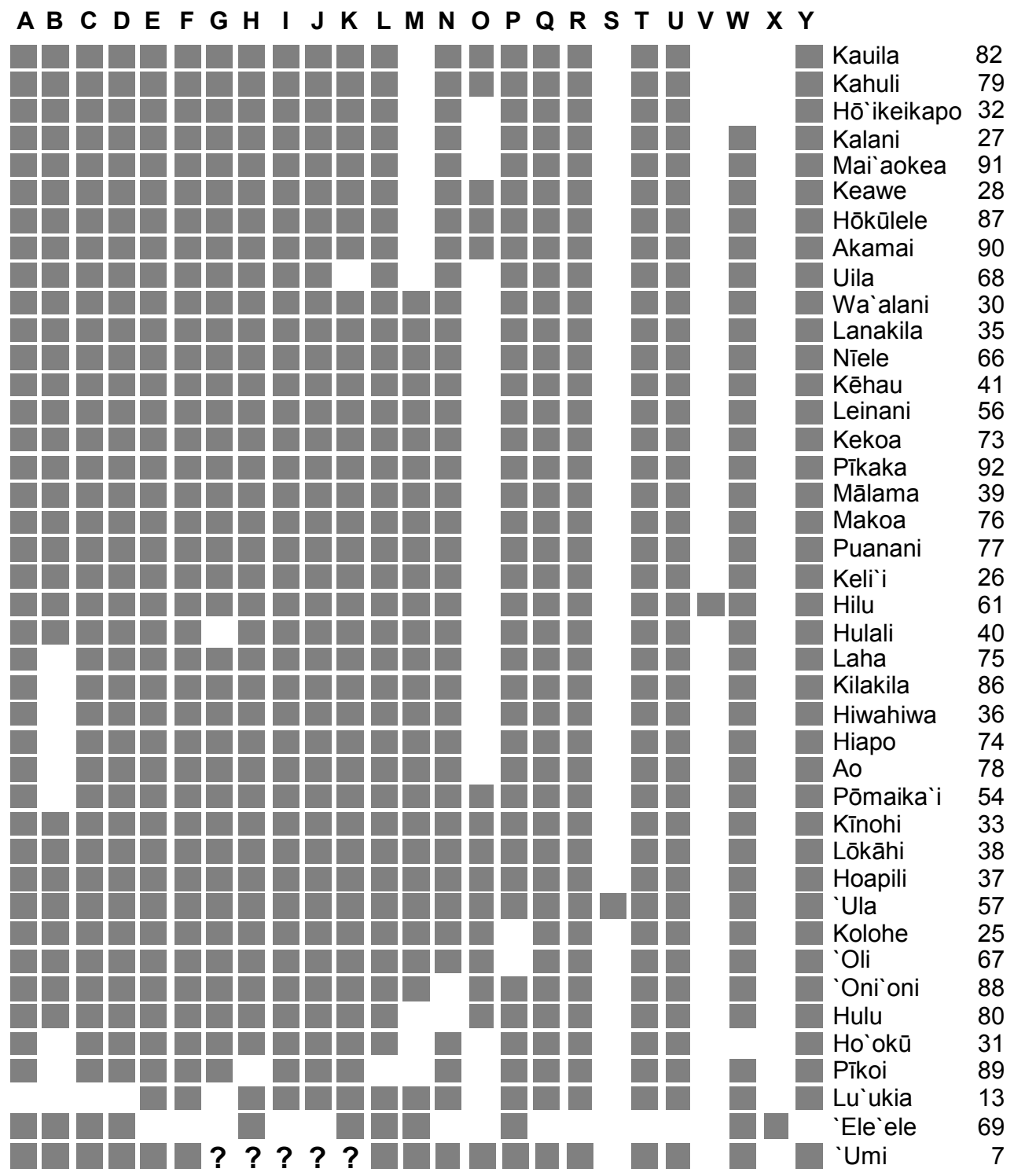

*Band Class Key for Figure 3 and all following Figures and Tables.

$\begin{array}{llll}\mathrm{A}=\mathrm{M} 3.118 & \mathrm{H}=\mathrm{M} 57.79 & \mathrm{O}=\mathrm{M} 60.306 & \mathrm{~V}=\mathrm{M} 62.68 \\ \mathrm{~B}=\mathrm{M} 5.59 & \mathrm{I}=\mathrm{M} 57.144 & \mathrm{P}=\mathrm{M} 60.307 & \mathrm{~W}=\mathrm{M} 62.124 \\ \mathrm{C}=\mathrm{M} 5.118 & \mathrm{~J}=\mathrm{M} 57.194 & \mathrm{Q}=\mathrm{M} 60.313 & \mathrm{X}=\mathrm{M} 62.146 \\ \mathrm{D}=\mathrm{M} 5.249 & \mathrm{~K}=\mathrm{M} 57.225 & \mathrm{R}=\mathrm{M} 61.57 & \mathrm{Y}=\mathrm{M} 62.313 \\ \mathrm{E}=\mathrm{M} 55.115 & \mathrm{~L}=\mathrm{M} 60.90 & \mathrm{~S}=\mathrm{M} 61.81 & \\ \mathrm{~F}=\mathrm{M} 55.131 & \mathrm{M}=\mathrm{M} 60.103 & \mathrm{~T}=\mathrm{M} 61.99 & \\ \mathrm{G}=\mathrm{M} 57.58 & \mathrm{~N}=\mathrm{M} 60.104 & \mathrm{U}=\mathrm{M} 62.61 & \end{array}$


Table 4. Summary of pattern designation for 41 'Alala (including 'Umi) based on presence (1) or absence (0) of polymorphic bands. Band classes (A-Y) as in Figure 3. Identical individuals are coded with the same color. Samples needing further analyses are indicated by “?".

\begin{tabular}{|c|c|c|c|c|c|c|c|c|c|c|c|c|c|c|c|c|c|c|c|c|c|c|c|c|c|c|}
\hline Individual & Pattern & $\mathrm{A}$ & $\mathrm{B}$ & $\mathrm{C}$ & $\mathrm{D}$ & $E$ & $\mathrm{~F}$ & $\mathrm{G}$ & $\mathrm{H}$ & $\mathrm{I}$ & $\mathrm{J}$ & $\mathrm{K}$ & $\mathrm{L}$ & $\mathrm{M}$ & $\mathrm{N}$ & $\mathrm{O}$ & $\mathrm{P}$ & $\mathrm{Q}$ & $\mathrm{R}$ & $\mathrm{S}$ & $\mathrm{T}$ & $\mathrm{U}$ & $\mathrm{V}$ & $\mathrm{W}$ & $\mathrm{X}$ & $\mathrm{Y}$ \\
\hline Wa`alani & 1 & 1 & 1 & 1 & 1 & 1 & 1 & 1 & 1 & 1 & 1 & 1 & 1 & 1 & 1 & 0 & 1 & 1 & 1 & 0 & 1 & 1 & 0 & 1 & 0 & 1 \\
\hline Lanakila & 1 & 1 & 1 & 1 & 1 & 1 & 1 & 1 & 1 & 1 & 1 & 1 & 1 & 1 & 1 & 0 & 1 & 1 & 1 & 0 & 1 & 1 & 0 & 1 & 0 & 1 \\
\hline Nīele & 1 & 1 & 1 & 1 & 1 & 1 & 1 & 1 & 1 & 1 & 1 & 1 & 1 & 1 & 1 & 0 & 1 & 1 & 1 & 0 & 1 & 1 & 0 & 1 & 0 & 1 \\
\hline Kēhau & 1 & 1 & 1 & 1 & 1 & 1 & 1 & 1 & 1 & 1 & 1 & 1 & 1 & 1 & 1 & 0 & 1 & 1 & 1 & 0 & 1 & 1 & 0 & 1 & 0 & 1 \\
\hline Leinani & 1 & 1 & 1 & 1 & 1 & 1 & 1 & 1 & 1 & 1 & 1 & 1 & 1 & 1 & 1 & 0 & 1 & 1 & 1 & 0 & 1 & 1 & 0 & 1 & 0 & 1 \\
\hline Kekoa & 1 & 1 & 1 & 1 & 1 & 1 & 1 & 1 & 1 & 1 & 1 & 1 & 1 & 1 & 1 & 0 & 1 & 1 & 1 & 0 & 1 & 1 & 0 & 1 & 0 & 1 \\
\hline Pīkaka & 1 & 1 & 1 & 1 & 1 & 1 & 1 & 1 & 1 & 1 & 1 & 1 & 1 & 1 & 1 & 0 & 1 & 1 & 1 & 0 & 1 & 1 & 0 & 1 & 0 & 1 \\
\hline Mālama & 1 & 1 & 1 & 1 & 1 & 1 & 1 & 1 & 1 & 1 & 1 & 1 & 1 & 1 & 1 & 0 & 1 & 1 & 1 & 0 & 1 & 1 & 0 & 1 & 0 & 1 \\
\hline Makoa & 1 & 1 & 1 & 1 & 1 & 1 & 1 & 1 & 1 & 1 & 1 & 1 & 1 & 1 & 1 & 0 & 1 & 1 & 1 & 0 & 1 & 1 & 0 & 1 & 0 & 1 \\
\hline Puanani & 1 & 1 & 1 & 1 & 1 & 1 & 1 & 1 & 1 & 1 & 1 & 1 & 1 & 1 & 1 & 0 & 1 & 1 & 1 & 0 & 1 & 1 & 0 & 1 & 0 & 1 \\
\hline Keli'i & 1 & 1 & 1 & 1 & 1 & 1 & 1 & 1 & 1 & 1 & 1 & 1 & 1 & 1 & 1 & 0 & 1 & 1 & 1 & 0 & 1 & 1 & 0 & 1 & 0 & 1 \\
\hline Laha & 2 & 1 & 0 & 1 & 1 & 1 & 1 & 1 & 1 & 1 & 1 & 1 & 1 & 1 & 1 & 0 & 1 & 1 & 1 & 0 & 1 & 1 & 0 & 1 & 0 & 1 \\
\hline Kilakila & 2 & 1 & 0 & 1 & 1 & 1 & 1 & 1 & 1 & 1 & 1 & 1 & 1 & 1 & 1 & 0 & 1 & 1 & 1 & 0 & 1 & 1 & 0 & 1 & 0 & 1 \\
\hline Hiwahiwa & 2 & 1 & 0 & 1 & 1 & 1 & 1 & 1 & 1 & 1 & 1 & 1 & 1 & 1 & 1 & 0 & 1 & 1 & 1 & 0 & 1 & 1 & 0 & 1 & 0 & 1 \\
\hline Hiapo & 2 & 1 & 0 & 1 & 1 & 1 & 1 & 1 & 1 & 1 & 1 & 1 & 1 & 1 & 1 & 0 & 1 & 1 & 1 & 0 & 1 & 1 & 0 & 1 & 0 & 1 \\
\hline Ao & 2 & 1 & 0 & 1 & 1 & 1 & 1 & 1 & 1 & 1 & 1 & 1 & 1 & 1 & 1 & 0 & 1 & 1 & 1 & 0 & 1 & 1 & 0 & 1 & 0 & 1 \\
\hline Kīnohi & 3 & 1 & 1 & 1 & 1 & 1 & 1 & 1 & 1 & 1 & 1 & 1 & 1 & 1 & 1 & 1 & 1 & 1 & 1 & 0 & 1 & 1 & 0 & 1 & 0 & 1 \\
\hline Lōkāhi & 3 & 1 & 1 & 1 & 1 & 1 & 1 & 1 & 1 & 1 & 1 & 1 & 1 & 1 & 1 & 1 & 1 & 1 & 1 & 0 & 1 & 1 & 0 & 1 & 0 & 1 \\
\hline Hoapili & 3 & 1 & 1 & 1 & 1 & 1 & 1 & 1 & 1 & 1 & 1 & 1 & 1 & 1 & 1 & 1 & 1 & 1 & 1 & 0 & 1 & 1 & 0 & 1 & 0 & 1 \\
\hline Umi & $3 ?$ & 1 & 1 & 1 & 1 & 1 & 1 & $?$ & $?$ & $?$ & $?$ & $?$ & 1 & 1 & 1 & 1 & 1 & 1 & 1 & 0 & 1 & 1 & 0 & 1 & 0 & 1 \\
\hline Keawe & 4 & 1 & 1 & 1 & 1 & 1 & 1 & 1 & 1 & 1 & 1 & 1 & 1 & 0 & 1 & 1 & 1 & 1 & 1 & 0 & 1 & 1 & 0 & 1 & 0 & 1 \\
\hline Hōkūlele & 4 & 1 & 1 & 1 & 1 & 1 & 1 & 1 & 1 & 1 & 1 & 1 & 1 & 0 & 1 & 1 & 1 & 1 & 1 & 0 & 1 & 1 & 0 & 1 & 0 & 1 \\
\hline Akamai & 4 & 1 & 1 & 1 & 1 & 1 & 1 & 1 & 1 & 1 & 1 & 1 & 1 & 0 & 1 & 1 & 1 & 1 & 1 & 0 & 1 & 1 & 0 & 1 & 0 & 1 \\
\hline Kalani & 5 & 1 & 1 & 1 & 1 & 1 & 1 & 1 & 1 & 1 & 1 & 1 & 1 & 0 & 1 & 0 & 1 & 1 & 1 & 0 & 1 & 1 & 0 & 1 & 0 & 1 \\
\hline Mai’aokea & 5 & 1 & 1 & 1 & 1 & 1 & 1 & 1 & 1 & 1 & 1 & 1 & 1 & 0 & 1 & 0 & 1 & 1 & 1 & 0 & 1 & 1 & 0 & 1 & 0 & 1 \\
\hline
\end{tabular}




\begin{tabular}{|c|c|c|c|c|c|c|c|c|c|c|c|c|c|c|c|c|c|c|c|c|c|c|c|c|c|c|}
\hline Kauila & 6 & 1 & 1 & 1 & 1 & 1 & 1 & 1 & 1 & 1 & 1 & 1 & 1 & 0 & 1 & 1 & 1 & 1 & 1 & 0 & 1 & 1 & 0 & 0 & 0 & 1 \\
\hline Kahuli & 6 & 1 & 1 & 1 & 1 & 1 & 1 & 1 & 1 & 1 & 1 & 1 & 1 & 0 & 1 & 1 & 1 & 1 & 1 & 0 & 1 & 1 & 0 & 0 & 0 & 1 \\
\hline Kolohe & 7 & 1 & 1 & 1 & 1 & 1 & 1 & 1 & 1 & 1 & 1 & 1 & 1 & 1 & 1 & 1 & 0 & 1 & 1 & 0 & 1 & 1 & 0 & 1 & 0 & 1 \\
\hline Oli & 7 & 1 & 1 & 1 & 1 & 1 & 1 & 1 & 1 & 1 & 1 & 1 & 1 & 1 & 1 & 1 & 0 & 1 & 1 & 0 & 1 & 1 & 0 & 1 & 0 & 1 \\
\hline Hula & 8 & 1 & 1 & 1 & 1 & 1 & 1 & 1 & 1 & 1 & 1 & 1 & 1 & 0 & 0 & 1 & 1 & 1 & 1 & 0 & 1 & 1 & 0 & 1 & 0 & 1 \\
\hline Hō ikeikapo & 9 & 1 & 1 & 1 & 1 & 1 & 1 & 1 & 1 & 1 & 1 & 1 & 1 & 0 & 1 & 0 & 1 & 1 & 1 & 0 & 1 & 1 & 0 & 0 & 0 & 1 \\
\hline Uila & 10 & 1 & 1 & 1 & 1 & 1 & 1 & 1 & 1 & 1 & 1 & 0 & 1 & 0 & 1 & 0 & 1 & 1 & 1 & 0 & 1 & 1 & 0 & 1 & 0 & 1 \\
\hline Oni`oni & 11 & 1 & 1 & 1 & 1 & 1 & 1 & 1 & 1 & 1 & 1 & 1 & 1 & 1 & 0 & 1 & 1 & 1 & 1 & 0 & 1 & 1 & 0 & 1 & 0 & 1 \\
\hline Ula & 12 & 1 & 1 & 1 & 1 & 1 & 1 & 1 & 1 & 1 & 1 & 1 & 1 & 1 & 1 & 1 & 1 & 1 & 1 & 1 & 1 & 1 & 0 & 1 & 0 & 1 \\
\hline Pōmaika`i & 13 & 1 & 0 & 1 & 1 & 1 & 1 & 1 & 1 & 1 & 1 & 1 & 1 & 1 & 1 & 1 & 1 & 1 & 1 & 0 & 1 & 1 & 0 & 1 & 0 & 1 \\
\hline Hilu & 14 & 1 & 1 & 1 & 1 & 1 & 1 & 1 & 1 & 1 & 1 & 1 & 1 & 1 & 1 & 0 & 1 & 1 & 1 & 0 & 1 & 1 & 1 & 1 & 0 & 1 \\
\hline Hulali & 15 & 1 & 1 & 1 & 1 & 1 & 1 & 0 & 1 & 1 & 1 & 1 & 1 & 1 & 1 & 0 & 1 & 1 & 1 & 0 & 1 & 1 & 0 & 1 & 0 & 1 \\
\hline Ho`okū & 16 & 1 & 0 & 1 & 1 & 1 & 1 & 1 & 1 & 1 & 1 & 1 & 1 & 0 & 1 & 0 & 1 & 1 & 1 & 0 & 1 & 1 & 0 & 0 & 0 & 1 \\
\hline Pīkoi & 17 & 1 & 0 & 1 & 1 & 1 & 1 & 1 & 1 & 1 & 1 & 1 & 0 & 0 & 1 & 0 & 1 & 1 & 1 & 0 & 1 & 1 & 0 & 1 & 0 & 1 \\
\hline Lu’ukia & 18 & 0 & 0 & 0 & 0 & 1 & 1 & 0 & 1 & 1 & 1 & 1 & 1 & 1 & 1 & 0 & 1 & 1 & 1 & 0 & 1 & 1 & 0 & 1 & 0 & 1 \\
\hline `Ele`ele & 19 & 1 & 1 & 1 & 1 & 0 & 0 & 0 & 1 & 0 & 0 & 1 & 1 & 1 & 0 & 0 & 1 & 0 & 0 & 0 & 0 & 0 & 0 & 1 & 1 & 0 \\
\hline
\end{tabular}


Table 5. Banding patterns for 41 captive `Alala summarized within families. Sire and Dam are the first two lines of each family group. Patterns are indicated and identical individuals are color coded as in Table 4. *Indicates possible pedigree discrepancy.

\begin{tabular}{|c|c|c|c|c|c|c|c|c|c|c|c|c|c|c|c|c|c|c|c|c|c|c|c|c|c|c|}
\hline & Pattern & $\mathrm{A}$ & B & $\mathrm{C}$ & $\mathrm{D}$ & $E$ & $F$ & $\mathrm{G}$ & $\mathrm{H}$ & $\mathrm{I}$ & $\mathrm{J}$ & $\mathrm{K}$ & $\mathrm{L}$ & $\mathrm{M}$ & $\mathrm{N}$ & $\mathrm{O}$ & $\mathrm{P}$ & $\mathrm{Q}$ & $\mathrm{R}$ & $\mathrm{S}$ & $\mathrm{T}$ & $\mathrm{U}$ & $\mathrm{V}$ & $\mathrm{W}$ & $X$ & $\mathrm{Y}$ \\
\hline \multicolumn{27}{|l|}{ Family 1} \\
\hline 'Umi & $3 ?$ & 1 & 1 & 1 & 1 & 1 & 1 & $?$ & $?$ & $?$ & $?$ & $?$ & 1 & 1 & 1 & 1 & 1 & 1 & 1 & 0 & 1 & 1 & 0 & 1 & 0 & 1 \\
\hline Lu’ukia & 18 & 0 & 0 & 0 & 0 & 1 & 1 & 0 & 1 & 1 & 1 & 1 & 1 & 1 & 1 & 0 & 1 & 1 & 1 & 0 & 1 & 1 & 0 & 1 & 0 & 1 \\
\hline Kelìi & 1 & 1 & 1 & 1 & 1 & 1 & 1 & 1 & 1 & 1 & 1 & 1 & 1 & 1 & 1 & 0 & 1 & 1 & 1 & 0 & 1 & 1 & 0 & 1 & 0 & 1 \\
\hline Kalani & 5 & 1 & 1 & 1 & 1 & 1 & 1 & 1 & 1 & 1 & 1 & 1 & 1 & 0 & 1 & 0 & 1 & 1 & 1 & 0 & 1 & 1 & 0 & 1 & 0 & 1 \\
\hline Keawe & 4 & 1 & 1 & 1 & 1 & 1 & 1 & 1 & 1 & 1 & 1 & 1 & 1 & 0 & 1 & 1 & 1 & 1 & 1 & 0 & 1 & 1 & 0 & 1 & 0 & 1 \\
\hline
\end{tabular}

Family 2

\begin{tabular}{llllllllllllllllllllllllllllll} 
Kalani & 5 & 1 & 1 & 1 & 1 & 1 & 1 & 1 & 1 & 1 & 1 & 1 & 1 & 0 & 1 & 0 & 1 & 1 & 1 & 0 & 1 & 1 & 0 & 1 & 0 & 1 \\
\hline Lu`ukia & 18 & 0 & 0 & 0 & 0 & 1 & 1 & 0 & 1 & 1 & 1 & 1 & 1 & 1 & 1 & 0 & 1 & 1 & 1 & 0 & 1 & 1 & & 0 & 1 & 0 & 1 \\
Ho`oku & 16 & 1 & 0 & 1 & 1 & 1 & 1 & 1 & 1 & 1 & 1 & 1 & 1 & 0 & 1 & 0 & 1 & 1 & 1 & 0 & 1 & 1 & & 0 & 0 & 0 & 1 \\
Hō'ikeikapo & 9 & 1 & 1 & 1 & 1 & 1 & 1 & 1 & 1 & 1 & 1 & 1 & 1 & 0 & 1 & 0 & 1 & 1 & 1 & 0 & 1 & 1 & 0 & 0 & 0 & 1
\end{tabular}

Family 3

\begin{tabular}{llllllllllllllllllllllllllllllll} 
Kalani & 5 & 1 & 1 & 1 & 1 & 1 & 1 & 1 & 1 & 1 & 1 & 1 & 1 & 0 & 1 & 0 & 1 & 1 & 1 & 0 & 1 & 1 & 0 & 1 & 0 & 1 \\
\hline 'Ula & 12 & 1 & 1 & 1 & 1 & 1 & 1 & 1 & 1 & 1 & 1 & 1 & 1 & 1 & 1 & 1 & 1 & 1 & 1 & 1 & 1 & 1 & & 0 & 1 & 0 & 1 \\
Laha & 2 & 1 & 0 & 1 & 1 & 1 & 1 & 1 & 1 & 1 & 1 & 1 & 1 & 1 & 1 & 0 & 1 & 1 & 1 & 0 & 1 & 1 & & 0 & 1 & 0 & 1 \\
Hiapo & 2 & 1 & 0 & 1 & 1 & 1 & 1 & 1 & 1 & 1 & 1 & 1 & 1 & 1 & 1 & 0 & 1 & 1 & 1 & 0 & 1 & 1 & 0 & 1 & 0 & 1
\end{tabular}

Family 4

$\begin{array}{llllllllllllllllllllllllllllllll}\text { Ho`oku } & 16 & 1 & 0 & 1 & 1 & 1 & 1 & 1 & 1 & 1 & 1 & 1 & 1 & 0 & 1 & 0 & 1 & 1 & 1 & 0 & 1 & 1 & 0 & 0 & 0 & 1 \\ \text { Keawe } & 4 & 1 & 1 & 1 & 1 & 1 & 1 & 1 & 1 & 1 & 1 & 1 & 1 & 0 & 1 & 1 & 1 & 1 & 1 & 0 & 1 & 1 & & 0 & 1 & 0 & 1 \\ \text { Kahuli } & 6 & 1 & 1 & 1 & 1 & 1 & 1 & 1 & 1 & 1 & 1 & 1 & 1 & 0 & 1 & 1 & 1 & 1 & 1 & 0 & 1 & 1 & 0 & 0 & 0 & 1 \\ \text { Kauila } & 6 & 1 & 1 & 1 & 1 & 1 & 1 & 1 & 1 & 1 & 1 & 1 & 1 & 0 & 1 & 1 & 1 & 1 & 1 & 0 & 1 & 1 & 0 & 0 & 0 & 1 \\ \text { Hokule } & 4 & 1 & 1 & 1 & 1 & 1 & 1 & 1 & 1 & 1 & 1 & 1 & 1 & 0 & 1 & 1 & 1 & 1 & 1 & 0 & 1 & 1 & 0 & 1 & 0 & 1 \\ \text { Oni`oni } & 11 & 1 & 1 & 1 & 1 & 1 & 1 & 1 & 1 & 1 & 1 & 1 & 1 & 1 * & 0 & 1 & 1 & 1 & 1 & 0 & 1 & 1 & 0 & 1 & 0 & 1\end{array}$


Pīkoi $\begin{array}{lllllllllllllllllllllllllll}17 & 1 & 0 & 1 & 1 & 1 & 1 & 1 & 1 & 1 & 1 & 1 & 0 & 0 & 1 & 0 & 1 & 1 & 1 & 0 & 1 & 1 & 0 & 1 & 0 & 1 \\ 4 & 1 & 1 & 1 & 1 & 1 & 1 & 1 & 1 & 1 & 1 & 1 & 1 & 0 & 1 & 1 & 1 & 1 & 1 & 0 & 1 & 1 & & 0 & 1 & 0 & 1\end{array}$

Family 5

\begin{tabular}{llllllllllllllllllllllllllllll} 
Hō'ikeikapo & 9 & 1 & 1 & 1 & 1 & 1 & 1 & 1 & 1 & 1 & 1 & 1 & 1 & 0 & 1 & 0 & 1 & 1 & 1 & 0 & 1 & 1 & 0 & 0 & 0 & 1 \\
\hline Wa`alani & 1 & 1 & 1 & 1 & 1 & 1 & 1 & 1 & 1 & 1 & 1 & 1 & 1 & 1 & 1 & 0 & 1 & 1 & 1 & 0 & 1 & 1 & 0 & 1 & 0 & 1 \\
Lanakila & 1 & 1 & 1 & 1 & 1 & 1 & 1 & 1 & 1 & 1 & 1 & 1 & 1 & 1 & 1 & 0 & 1 & 1 & 1 & 0 & 1 & 1 & & 0 & 1 & 0 & 1 \\
Nīele & 1 & 1 & 1 & 1 & 1 & 1 & 1 & 1 & 1 & 1 & 1 & 1 & 1 & 1 & 1 & 0 & 1 & 1 & 1 & 0 & 1 & 1 & 0 & 1 & 0 & 1 \\
Kekoa & 1 & 1 & 1 & 1 & 1 & 1 & 1 & 1 & 1 & 1 & 1 & 1 & 1 & 1 & 1 & 0 & 1 & 1 & 1 & 0 & 1 & 1 & & 0 & 1 & 0 & 1 \\
Makoa & 1 & 1 & 1 & 1 & 1 & 1 & 1 & 1 & 1 & 1 & 1 & 1 & 1 & 1 & 1 & 0 & 1 & 1 & 1 & 0 & 1 & 1 & 0 & 1 & 0 & 1 \\
Puanani & 1 & 1 & 1 & 1 & 1 & 1 & 1 & 1 & 1 & 1 & 1 & 1 & 1 & 1 & 1 & 0 & 1 & 1 & 1 & 0 & 1 & 1 & 0 & 1 & 0 & 1 \\
Pīkaka & 1 & 1 & 1 & 1 & 1 & 1 & 1 & 1 & 1 & 1 & 1 & 1 & 1 & 1 & 1 & 0 & 1 & 1 & 1 & 0 & 1 & 1 & 0 & 1 & 0 & 1 \\
Mai’aokea & 5 & 1 & 1 & 1 & 1 & 1 & 1 & 1 & 1 & 1 & 1 & 1 & 1 & 0 & 1 & 0 & 1 & 1 & 1 & 0 & 1 & 1 & 0 & 1 & 0 & 1 \\
Ao & 2 & 1 & 0 & 1 & 1 & 1 & 1 & 1 & 1 & 1 & 1 & 1 & 1 & 1 & 1 & 0 & 1 & 1 & 1 & 0 & 1 & 1 & 0 & 1 & 0 & 1 \\
Kilakila & 2 & 1 & 0 & 1 & 1 & 1 & 1 & 1 & 1 & 1 & 1 & 1 & 1 & 1 & 1 & 0 & 1 & 1 & 1 & 0 & 1 & 1 & 0 & 1 & 0 & 1 \\
\hline Uila & 10 & 1 & 1 & 1 & 1 & 1 & 1 & 1 & 1 & 1 & 1 & 0 & 1 & 0 & 1 & 0 & 1 & 1 & 1 & 0 & 1 & 1 & 0 & 1 & 0 & 1 \\
'Ele`ele & 19 & 1 & 1 & 1 & 1 & 0 & 0 & 0 & 1 & 0 & 0 & 1 & 1 & 1 & 0 & 0 & 1 & 0 & 0 & 0 & 0 & 0 & 0 & 1 & $1 *$ & 0
\end{tabular}

Family 6 Kalāhiki pair

\begin{tabular}{|c|c|c|c|c|c|c|c|c|c|c|c|c|c|c|c|c|c|c|c|c|c|c|c|c|c|c|}
\hline Hiwahiwa & 2 & 1 & 0 & 1 & 1 & 1 & 1 & 1 & 1 & 1 & 1 & 1 & 1 & 1 & 1 & 0 & 1 & 1 & 1 & 0 & 1 & 1 & 0 & 1 & 0 & 1 \\
\hline Lōkāhi & 3 & 1 & 1 & 1 & 1 & 1 & 1 & 1 & 1 & 1 & 1 & 1 & 1 & 1 & 1 & 1 & 1 & 1 & 1 & 0 & 1 & 1 & 0 & 1 & 0 & 1 \\
\hline Hoapili & 3 & 1 & 1 & 1 & 1 & 1 & 1 & 1 & 1 & 1 & 1 & 1 & 1 & 1 & 1 & 1 & 1 & 1 & 1 & 0 & 1 & 1 & 0 & 1 & 0 & 1 \\
\hline Lōkāhi & 3 & 1 & 1 & 1 & 1 & 1 & 1 & 1 & 1 & 1 & 1 & 1 & 1 & 1 & 1 & 1 & 1 & 1 & 1 & 0 & 1 & 1 & 0 & 1 & 0 & 1 \\
\hline ‘Ula & 12 & 1 & 1 & 1 & 1 & 1 & 1 & 1 & 1 & 1 & 1 & 1 & 1 & 1 & 1 & 1 & 1 & 1 & 1 & $1^{*}$ & 1 & 1 & 0 & 1 & 0 & 1 \\
\hline Leinani & 1 & 1 & 1 & 1 & 1 & 1 & 1 & 1 & 1 & 1 & 1 & 1 & 1 & 1 & 1 & 0 & 1 & 1 & 1 & 0 & 1 & 1 & 0 & 1 & 0 & 1 \\
\hline
\end{tabular}

Family 7 Keālia pair

\begin{tabular}{llllllllllllllllllllllllllllllll} 
Mālama & 1 & 1 & 1 & 1 & 1 & 1 & 1 & 1 & 1 & 1 & 1 & 1 & 1 & 1 & 1 & 0 & 1 & 1 & 1 & 0 & 1 & 1 & 0 & 1 & 0 & 1 \\
Kēhau & 1 & 1 & 1 & 1 & 1 & 1 & 1 & 1 & 1 & 1 & 1 & 1 & 1 & 1 & 1 & 0 & 1 & 1 & 1 & 0 & 1 & 1 & 0 & 1 & 0 & 1 \\
Hulali & 15 & 1 & 1 & 1 & 1 & 1 & 1 & 0 & 1 & 1 & 1 & 1 & 1 & 1 & 1 & 0 & 1 & 1 & 1 & 0 & 1 & 1 & 0 & 1 & 0 & 1 \\
\hline
\end{tabular}


Evaluation of family relationships can be addressed using AFLP data when both parents are homozygous for the absence of alleles $(0 / 0)$ and offspring show a presence of alleles $(+/+$ or $+/ 0)$. This occurred in three instances (indicated by "*" in Table 5). One is the detection of a band at locus M60.103 in progeny of 'Oni'oni, when parents Ho`okū and Keawe are both homozygous 0/0. The second is at locus M62.146, where 'Ele'ele possesses this band, while neither parent does (Hō'ikeikapo and Wa'alani), and third at locus m61.81 where 'Ula possesses this band and both parents (Hiwahiwa and Lōkāhi) do not. The corresponding microsatellite data submitted by Rob Fleischer do not validate or refute these data. The microsatellite data indicate other possible discrepancies (Kinnohi, Keli'i, and Uila) that the AFLP data neither validate nor refute. The microsatellite data also suggests that extra-pair fertilization resulted in the observance of 5 alleles at one locus MJG1 in the Keâlia pair. The AFLP data show no abnormalities in that family (although the parents are not available for testing). Additional analyses are required to definitively resolve these discrepancies.

Within-family analyses can shed light on individual level of heterozygosity. In the instances where at least one of the offspring are $(0 / 0)$ at a particular locus, and either or both parents are $(+)$, the $(+)$ parent(s) can be defined as being heterozygous at that particular locus. This occurs in 2/25 loci in Family 1 (0.08), 3/25 in Families 2 and 3 (0.12), 5/25 in Family 4 (0.2), and 14/25 in Family 5 (0.56) (Table 5). Frequency of polymorphic loci and corresponding levels of heterozygocity among parents Hō'ikeikapo and Wa alani in Family 5 appear to be greater than in other families that were evaluated. However, 11/14 of these polymorphic loci in Family 5 are due to the absence of a band in only one individual, 'Ele'ele, where all other siblings were scored as present for these bands. 'Ele'ele appears very distinct from siblings. We obtained similar results when the sample was repeated. Further work to confirm these differences would be useful.

It is not possible to directly distinguish heterozygotes (+/-) from homozygotes $(+/+)$ at the population level because of the dominant nature of AFLP, but presence or absence data can be converted to expected heterozygosity estimates which are then comparable to those determined by codominant markers (e.g., microsatellites). Most of these estimates are calculated using the assumptions of the standard random-mating model (Hardy-Weinberg equilibrium). The average expected heterozygosity $\left(\mathrm{H}_{0}=1-\mathrm{q} 2-(1-\mathrm{q}) 2\right)$ for each AFLP locus is presented in Table 6 (Hartl and Clark 1997). Two average values of $\mathrm{H}_{0}$ are listed. One (inclusive) is the average $\mathrm{H}_{0}$ for all loci regardless of the number of homozygous absence individuals (-/-) at each locus. There have been concerns regarding bias introduced when calculating small values of q (frequency of absence allele). This bias is nearly eliminated when only loci that have 4 or more individuals who are homozygous for the absence allele (-/-) are included in the analyses. Another method applies Bayesian statistics to estimate the frequency of the absence allele (q) that nearly eliminates all bias and does not assume that genotypes within populations are in Hardy-Weinberg equilibrium (Zhivotovsky 1999; Holsinger et al., 2002). We have included an average $\mathrm{H}_{0}$ using only the loci with 4 or more (-/-) individuals as well as the Bayesian estimate generated in the program Hickory (v.1.0.3). Our $\mathrm{H}_{0}$ estimate range of 0.229-0.265 (Table 6) based on 25 polymorphic loci is in the range of the estimates provided by the microsatellite data from 5 loci (0.248) (excluding the MJG1 locus) as reported by Fleischer (2003). It is much lower than the average $\mathrm{H}_{0}$ estimate provided from 6 microsatellite loci $(0.319)$ when the MJG1 locus is included. The statistic $F_{I S}$ is the inbreeding coefficient of a group of inbred organisms relative to the subpopulation to which they belong. The statistic $\theta^{\mathrm{B}}=$ Fst which is the reduction in heterozygosity due to increased inbreeding 
relative to the total population. These low statistical estimates (Table 6) are indicative of a highly inbred population.

Table 6. Heterozygosity $\left(\mathrm{H}_{0}\right)$ at each locus

\begin{tabular}{lllllllll}
\hline Locus & $\mathrm{n}$ & $\mathrm{A}$ & $\mathrm{R}$ & $\mathrm{q}$ & $\mathrm{p}$ & $\mathrm{p} 2$ & $\mathrm{q} 2$ & $\mathrm{H} 0$ \\
\hline M3.118 & 41 & 1 & 0.24 & 0.156 & 0.844 & 0.712 & 0.024 & 0.263 \\
M5.59 & 41 & 9 & 0.219 & 0.468 & 0.532 & 0.283 & 0.219 & 0.498 \\
M5.118 & 41 & 1 & 0.24 & 0.156 & 0.844 & 0.712 & 0.024 & 0.263 \\
M5.249 & 41 & 1 & 0.24 & 0.156 & 0.844 & 0.712 & 0.024 & 0.263 \\
M55.115 & 41 & 1 & 0.24 & 0.156 & 0.844 & 0.712 & 0.024 & 0.263 \\
M55.131 & 41 & 1 & 0.24 & 0.156 & 0.844 & 0.712 & 0.024 & 0.263 \\
M60.90 & 41 & 1 & 0.24 & 0.156 & 0.844 & 0.712 & 0.024 & 0.263 \\
M60.103 & 41 & 12 & 0.293 & 0.541 & 0.459 & 0.211 & 0.293 & 0.497 \\
M60.104 & 41 & 3 & 0.073 & 0.271 & 0.729 & 0.531 & 0.073 & 0.395 \\
M60.306 & 41 & 26 & 0.634 & 0.796 & 0.204 & 0.042 & 0.634 & 0.325 \\
M60.307 & 41 & 2 & 0.049 & 0.22 & 0.78 & 0.608 & 0.048 & 0.343 \\
M60.313 & 41 & 1 & 0.24 & 0.156 & 0.844 & 0.712 & 0.024 & 0.263 \\
M61.57 & 41 & 1 & 0.24 & 0.156 & 0.844 & 0.712 & 0.024 & 0.263 \\
M61.81 & 41 & 40 & 0.976 & 0.987 & 0.013 & 0.00017 & 0.974 & 0.026 \\
M61.99 & 41 & 1 & 0.24 & 0.156 & 0.844 & 0.712 & 0.024 & 0.263 \\
M62.61 & 41 & 1 & 0.24 & 0.156 & 0.844 & 0.712 & 0.024 & 0.263 \\
M62.68 & 41 & 40 & 0.976 & 0.987 & 0.013 & 0.00017 & 0.974 & 0.026 \\
M62.124 & 41 & 4 & 0.096 & 0.312 & 0.688 & 0.473 & 0.097 & 0.429 \\
M62.146 & 41 & 40 & 0.976 & 0.987 & 0.013 & 0.00017 & 0.974 & 0.026 \\
M62.313 & 41 & 1 & 0.24 & 0.156 & 0.844 & 0.712 & 0.024 & 0.263 \\
M57.58 & 40 & 3 & 0.075 & 0.274 & 0.726 & 0.527 & 0.075 & 0.398 \\
M57.79 & 40 & 1 & 0.025 & 0.158 & 0.842 & 0.709 & 0.025 & 0.266 \\
M57.145 & 40 & 1 & 0.025 & 0.158 & 0.842 & 0.709 & 0.025 & 0.266 \\
M57.194 & 40 & 1 & 0.025 & 0.158 & 0.842 & 0.709 & 0.025 & 0.266 \\
M57.250 & 40 & 1 & 0.025 & 0.158 & 0.842 & 0.709 & 0.025 & 0.266 \\
\hline Mean $\mathrm{H}_{0}$ & & & & & & & & \\
inclusive & & & & & & & & 0.265 \\
Mean $\mathrm{H}_{0}$ & & & & & & & & \\
& & & & & & & & 0.229
\end{tabular}


A $>3$

Mean $\mathrm{H}_{0}$

Bayesian

fis

0.1352

$\theta^{\mathrm{B}}$

$\mathrm{n}=$ number of individuals evaluated

$A=$ number of individuals homozygous for absence alleles (-/-)

$\mathrm{R}=$ Frequency of homozygous recessives $(\mathrm{A} / \mathrm{n})$

$q=\sqrt{\mathrm{R}}$ (frequency of absence allele)

$\mathrm{H}_{0}=1-\mathrm{q} 2-(1-\mathrm{q}) 2$

fis = inbreeding coefficient of group of inbred organisms relative to the subpopulation to which they belong.

$\theta^{B}=$ fst, reduction in heterozygosity due to increased inbreeding relative to the total population.

Results of phylogenetic relationships are summarized using all 7 sets of primers (Figures 4 and 5, excluding founding individual 'Umi) or using only 6 sets of primers (Figures 6 and 7, including 'Umi). In Figures 6 and 7, primer set M57 is excluded because it produced inconclusive results for 'Umi. Results are also presented as either all 255 polymorphic and monomorphic bands (Figures 4 and 6) or only the 35 polymorphic band classes (Figures 5 and 7). Relationships between individuals are depicted in UPGMA dendrograms derived from similarity values estimated by methods of Dice.

Results from analyses of all 7 sets of primers including both monomorphic and polymorphic bands are presented in Figure 4. 'Umi is not included (but is inserted in parentheses where he would be expected). Two distinct main clusters were generated from the data. All individuals originating from McCandless Ranch fall within the larger of the two clusters. Similarity values range from 97.2 to 100 . If monomorphic bands are removed from the analyses and only polymorphic bands are used, the same two basic clusters are generated based on similarity values, with 'Oni'oni now placed outside the larger cluster (containing the McCandless birds) with sibling 'Ula (from Ho 'okū and Keawe, Figure 5). To include 'Umi in phylogenetic analyses, we needed to remove one primer set (M57) in the analysis as DNA results from 'Umi need to be re-run with this primer set. With only 6 sets of primers, the very distinct clustering is lost, but the relationship of 'Umi is established (Figures 6 and 7). 
Figure 4. Relationships of 40 individuals ( Umi excluded) based on seven primer sets are depicted in a UPGMA dendrogram using distance estimates (Dice) calculated in the program Bionumerics. The individuals originating from McCandless Ranch (boxes) are contained within one cluster. All monomorphic and polymorphic bands $(\mathrm{n}=255)$ are included. Studbook number follows each name.

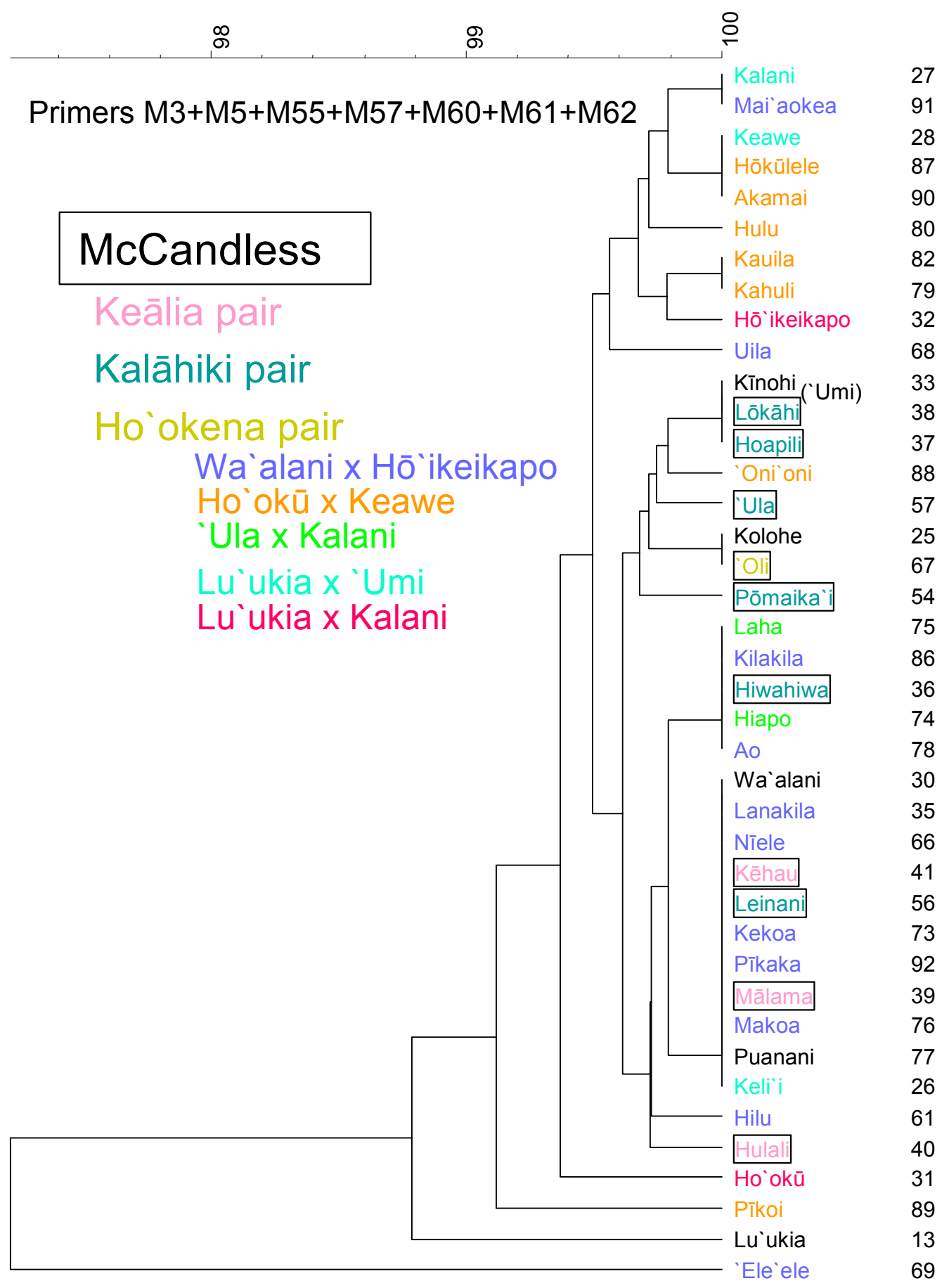


Figure 5. Relationships of 40 individuals based on seven primer sets ('Umi excluded) are depicted in a UPGMA dendrogram using distance estimates (Dice) calculated in the program Bionumerics. The individuals originating from McCandless Ranch are contained within one cluster. Only polymorphic bands $(n=25)$ are included. Studbook number follows each name.

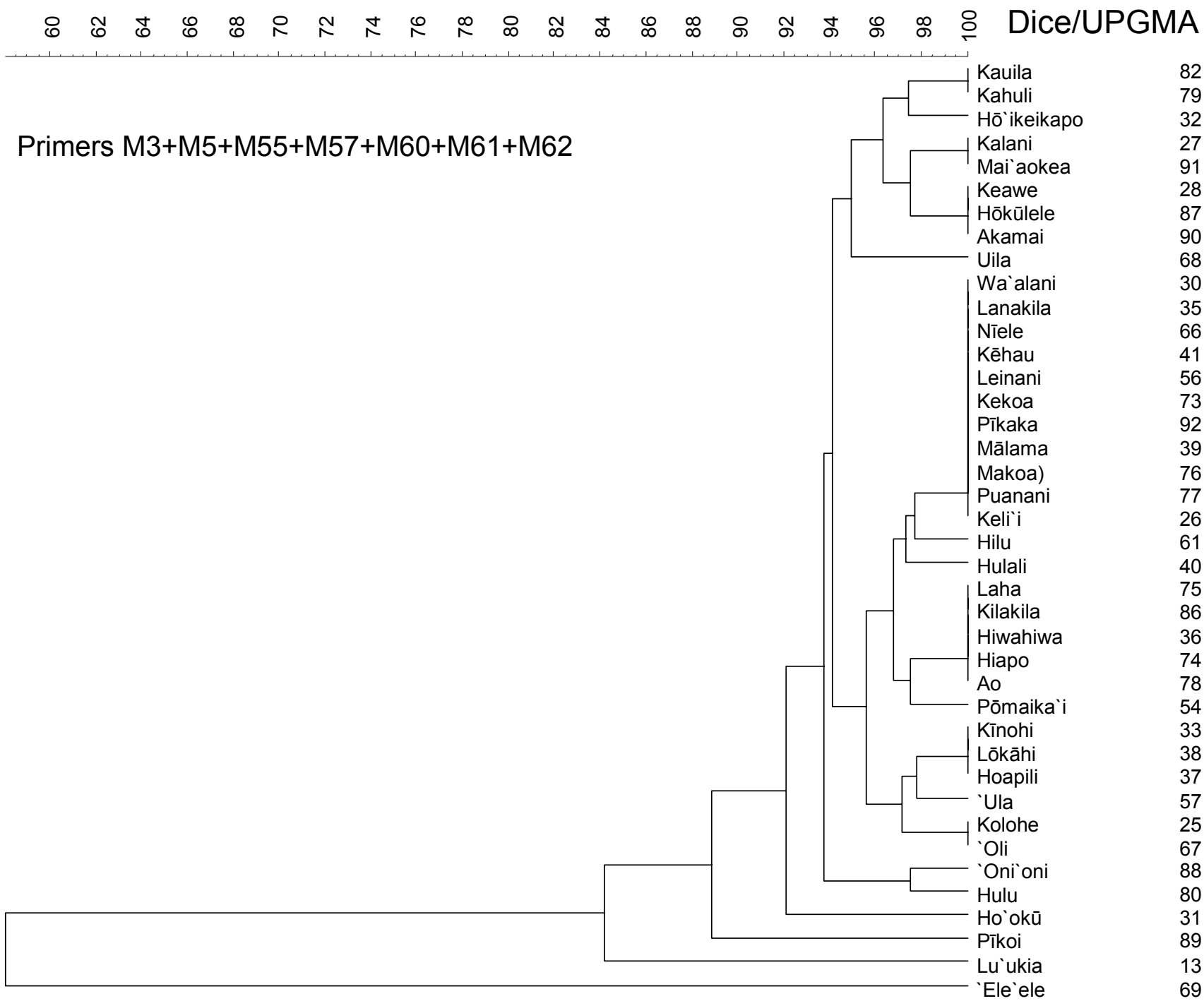


Figure 6. Relationships of 41 individuals (Umi included) based on six primer sets are depicted in a UPGMA dendrogram using distance estimates (Dice) calculated in the program Bionumerics. Birds originating from McCandless Ranch are designated by boxes. All monomorphic and polymorphic bands $(n=255)$ are included. Studbook number follows each name.

\section{Primers M3+M5+M55+M60+M61+M62}

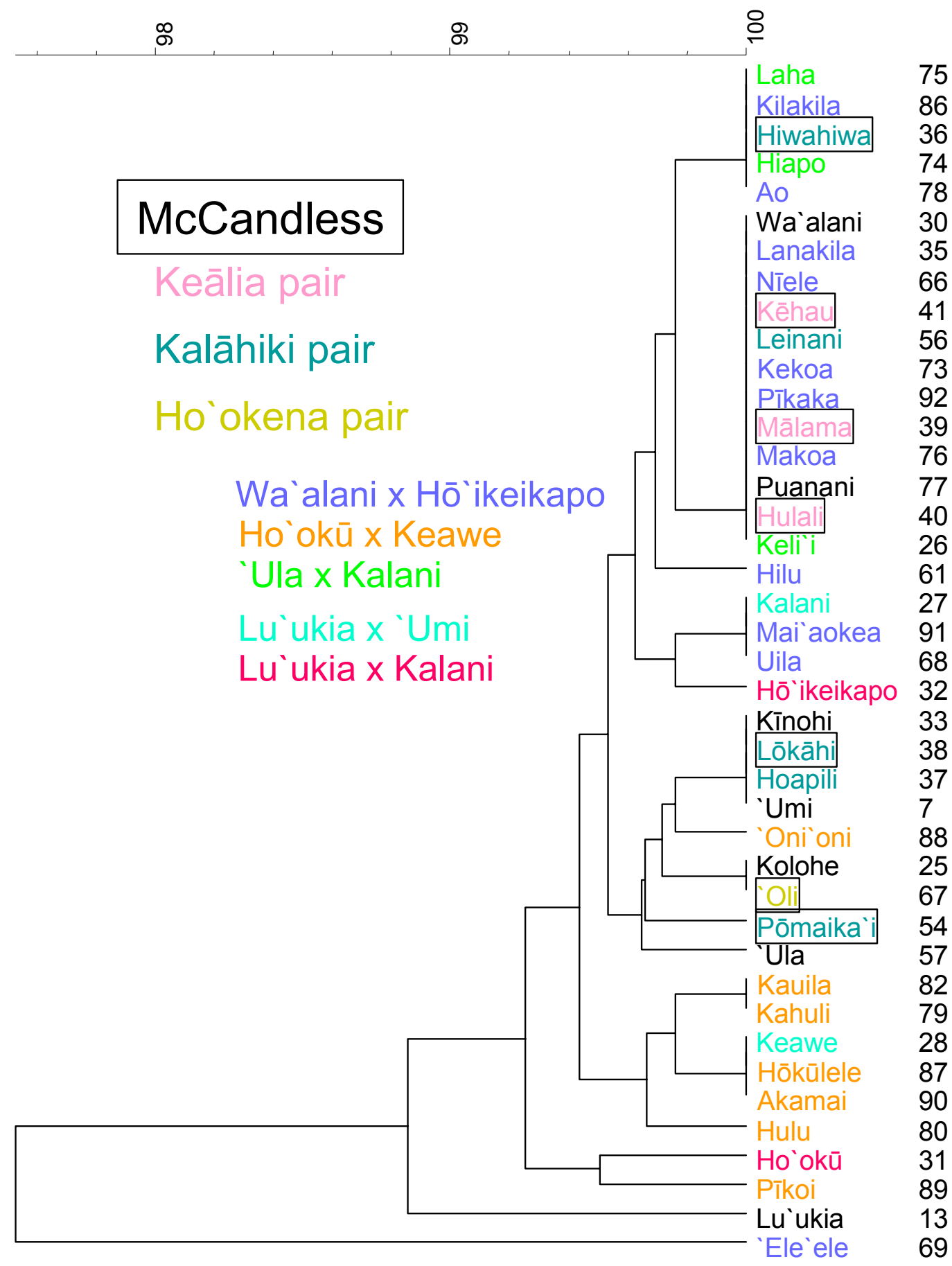


Figure 7. Relationships of 41 individuals (Umi included) based on six primer sets are depicted in a UPGMA dendrogram using distance estimates (Dice) calculated in the program Bionumerics.

Only polymorphic bands $(\mathrm{n}=25)$ are included in calculating distance estimates. Studbook number follows each name.

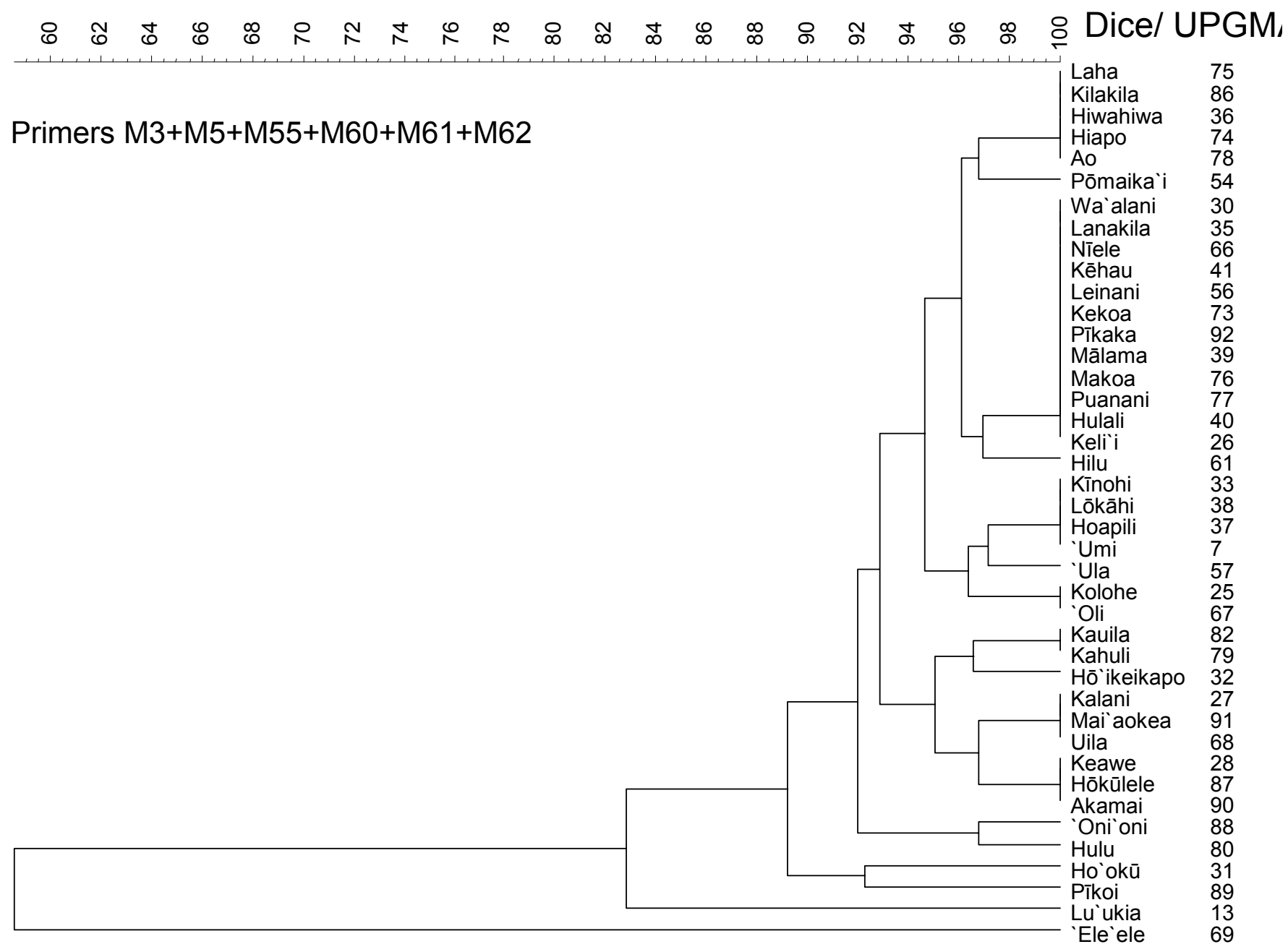


The similarity values among mating pairs are summarized in Table 7. Genetic distances between 'Umi and Lu'ukia and Kalani and Lu'ukia appear greater then others with lower similarity values of 82.76 and 83.33, respectively. Among founders (data not shown) based on only polymorphic bands 'Umi appears more similar to Wa'alani with a similarity value 96.97 than to mate Lu 'ukia (similarity value 82.76). Wa 'alani and Lu 'ukia have a similarity value of 85.71.

Table 7. Summary of similarity values among mating pairs. All bands/polymorphic bands only. All based on matrix generated using 7 sets of primers, except 'Umi x Lu ukia which was based on 6 sets (excluding M57). Studbook numbers follow each name.

\begin{tabular}{ccccc}
\hline & Hō`ikeikapo (32) & Keawe (28) & Kalani (27) & 'Umi (7) \\
\hline Wa`alani (30) & $99.58 / 95.00$ & & & \\
Ho`okū (31) & & $99.37 / 92.31$ & & \\
`Ula (57) & & & $99.37 / 93.02$ & \\
Lu`ukia (13) & & $98.73 / 83.33$ & $98.79 / 82.76$ \\
\hline
\end{tabular}

When comparing results from the 6 (20 polymorphic bands) versus 7 sets ( 25 polymorphic bands), the inclusion of primer set M57 provides $25 \%$ overall increased resolution. While much valuable information was obtained by these analyses, further additional analyses are needed to: (1) resolve discrepancies found by AFLP and microsatellite data and further clarify the pedigree, (2) repeat AFLP analyses to include all individuals available (including 2002-present), and (3) include at least 12 sets of primers to further resolve genetic distinctions among individuals. Based on numbers of polymorphic bands observed in other (non-inbred) species, we would estimate that 9-12 sets of primers should be included for a full analysis of this population.

\section{Summary}

AFLP (amplified fragment length polymorphism) is a universal technique for DNA fingerprinting. This study was undertaken to evaluate the level of genetic diversity in the captive population of 'Alalā by AFLP, to compare genetic data with currently available pedigree information, and to determine the extent of relatedness of mating pairs and among founding individuals. A total of seven primer sets and 41 individuals were used for final analyses. Bands produced by these 7 sets of primers are designated as either present (1) or absent (0). We evaluated a total of 255 loci for polymorphism and of these $9.8 \%$ (25) were polymorphic. Nineteen (19) distinct banding patterns were distinguished among these 41 individuals with Pattern 1 being the predominant pattern. This pattern includes individuals from several families and lineages. Evaluation of family relationships can be addressed using AFLP data when both parents are homozygous for the absence of alleles $(0 / 0)$ and offspring show a presence $(+/+$ or $+/ 0)$. This phenomenon occurred in three instances, however, parallel microsatellite data neither validate nor refute these observations. Heterozygosity values $\left(\mathrm{H}_{0}\right)$ were estimated based on the 25 polymorphic loci. Included are average $\mathrm{H}_{0}$ using only the loci with 4 or more (-/-) individuals, and the Bayesian estimate generated in the program Hickory (v.1.0.3). Our $\mathrm{H}_{0}$ estimate range of 0.2290.265 based on 25 polymorphic loci is in the range of estimates provided by microsatellite data 
from 5 loci (0.248) (excluding the MJG1 locus) as reported by Fleischer (2003). It is much lower than the average $\mathrm{H}_{0}$ estimate provided from 6 microsatellite loci $(0.319)$ when the MJG1 locus is included. The value of the use of additional primer sets is evident when comparing results using 6 (20 polymorphic bands) vs 7 sets ( 25 polymorphic bands). Inclusion of one additional primer set provides $25 \%$ overall increased resolution.

With all 7 sets of primers (Figure 3), two main clusters include 36 of the 40 (excluding 'Umi) birds analyzed, with all of the McCandless Ranch birds contained in one cluster. While overall diversity is quite low, it is recommended that when the option for selecting breeding pairs exists, managers should avoid selecting individuals with similar or identical banding patterns. No data are currently available on more recently hatched captive birds. While valuable information was obtained by these analyses, additional analyses are needed to: (1) resolve discrepancies found by AFLP and microsatellite data and further clarify the pedigree, (2) repeat AFLP analyses to attempt to include all individuals currently available, and (3) include additional sets of primers to further resolve genetic distinctions among individuals. Based on numbers of polymorphic bands observed in this species compared with other (non-inbred) species, at least 9-12 sets of primers should be included for a full analysis of this captive population.

\section{Acknowledgments}

This research was supported in part by the U.S. Fish and Wildlife Service, the USGS Pacific Island Ecosystems Research Center, the USGS Wildlife: Terrestrial and Endangered Resources Program and National Science Foundation grant DEB-0083944. Mahalo i ka po`e o Hawai'i no ko lākou kōkua a me ko lākou aloha. Thank you very much to the people of Hawai'i for their help and aloha.

\section{References Cited}

Bensch S, and M Akesson 2005, Ten years of AFLP in ecology and evolution: why so few animals? Molecular Ecology 14(10):2899-914.

Fleischer RC. 2003. Genetic analysis of captive 'alalā (Corvus hawaiiensis). Unpublished report to U. S. Fish and Wildlife Service.

Hartl DL and Clark AG. 1997, Principles of Population Genetics. 3rd edition. Sinaur Associates Inc. p34.

Holsinger KE, Lewis PO and Dey DK 2002, A Bayesian approach to inferring population structure from dominant markers. Molecular Ecology 11:1157-1164.

Sachidanandam R, Weissman D, Schmit SC et al., 2001, A map of human genome sequence variation containing 1.42 million single nucleotide polymorphisms. Nature 409:928-933.

Vos P., Hogers R., Bleeker M et al., 1995, A new technique for DNA fingerprinting. Nucleic Acids Research 23:4407-4414. 
Zhivotovsky LA 1999, Estimating population structure in diploids with multilocus dominant markers. Molecular Ecology 8:907-913. 


\section{Appendix}

\section{Details of AFLP procedures}

\section{$\underline{\text { Reference Codes for Eco x Mse primer combinations }}$}

\begin{tabular}{|c|c|c|}
\hline$\underline{\text { Primer Set }}$ & $\underline{E C O}$ & Mse \\
\hline M3 & ER1.P2 (ATA) & Mse.P45 (ATG) \\
\hline M5 & ER1.P4 (ACA) & Mse.P35 (ACA) \\
\hline M55 & ER1.P59 (CTA) & Mse.P51 (CCA) \\
\hline M57 & ER1.P59 (CTA) & Mse.P56 (CGC) \\
\hline M60 & ER1.P55 (CGA) & Mse.P56 (CGC) \\
\hline M61 & ER1.P49 (CAG) & Mse.P51 (CCA) \\
\hline M62 & ER1.P49 (CAG) & Mse.P53 (CCG) \\
\hline
\end{tabular}

\section{STEP \#1 - Band Classes and Scoring}

complete).

Scoring procedure for each primer set is as follows (after all gel input and normalizing is

1. Scan gel, observe both data lanes and ROX500 lanes to determine any problems with lanes, such as poor loading or poor reaction.

2. Using previously determined bands from primer screen gel, make an Excel spreadsheet to note which bands will be used or not, the range of bp (to 1 decimal pt) for each class, and if not used, why (i.e., shoulder, abnormal). Note the number of bands in original list from primer screen gel.

3. Score each gel for that primer set as described next, making any necessary changes to band classes used. It is most consistent to do the entire gel at one time, but within the gel one band at a time for all individuals on a gel will suffice.

4. Open comparison window with all individuals. Select the primer set and have the bands show. Stretch out the view area and turn off band class and turn it back on. Use same settings always used for band classes (optimization 1\%, position tolerance $0.16 \%$, others default 0 ). Proof each band class against Excel sheet from Step 1. Note \# bands and \# polymorphic bands.

5. Compare reruns of same individual (4 repeats, the gel reruns and 2 samples from extraction rerun) using band class table (green squares). Make notes in column 3 of Excel sheet as to which samples have inconsistencies for which band. (Note that repeats are labeled as 6933.13 for $6933 \mathrm{rpt} 13$ ). Re-examine each sample with inconsistencies. Highlight cell in column 3 pink if deciding not to use and make changes to gel and band class in comparison window. Decide which bands not to use based on comparing bands in lanes of reruns. Note new total \# bands and \# polymorphic bands. 
6. In comparison window, compare bands across family groups. Note which bands are not inherited

7. After all primer sets are scored for the first 4 Steps, remove individuals that should be rerun and recount the \# of bands.

\section{Primer set \#1 - M3}

Bad lanes:

Gel A10 03 (A10.01-A10.42):

Gel A10_04: On full exposure there is pull up on all samples, appears to be overflow from neighboring lanes from loading, throughout gel.

Quality of reaction: A10.09 is a bit light but usable

In preamp: The following lanes had very light reactions: A10.11, A10.12, A10.15, A10.16, A10.34, A10.40, and A10.41

Gel A15 03 (A15.15-A15.21):

Gel A15_04: unknown bands until after 250, OK

Quality of reaction: A15.18 is blank, unusable

In preamp: the following lanes had very light reactions: A15.06, A15.13, A15.20.

Gel A16 03 (A16.21-A16.30):

Gel A16_04: Lanes A16.22 and A16.24 have some pull up after 250, but light

Quality of reaction: All look good

In preamp: The following lane had very light reaction: A16.09, A16.19, A16.29.

Gel A17 03 (A17.25-A17.36):

Gel A17_04: A17.29+35 have pull-up at 185bp and A17.32+34 at 175bp and A17.29 at 235bp

Quality of reaction: A17.26 looks questionable

In preamp: A17.13-A17.15, A17.21-A17.23 all had light reactions, with A17.14, A17.15, and

A17.23 being the worst.

\# bands: 36

\# polymorphic bands: 19

\section{Primer set \#2 - M5}

Bad lanes:

Gel A10 02 (A10.01-A10.42):

Gel A10_04: On full exposure there is pull up on all samples, appears to be overflow from neighboring lanes from loading, throughout gel.

Quality of reaction: A10.09 is a bit light but usable

In preamp: The following lanes had very light reactions: A10.11, A10.12, A10.15, A10.16, A10.34, A 10.40 , and A10.41

Gel A15 02 (A15.15-A15.21):

Gel A15_04: Unknown bands until after 250, OK

Quality of reaction: A15.18 is blank, unusable

In preamp: The following lanes had very light reactions: A15.06, A15.13, A15.20.

Gel A16 02 (A16.21-A16.30): 
Gel A16_04: Lanes A16.22 and A16.24 have some pull-up after 250, but light

Quality of reaction: All look good

In preamp: The following lane had very light reaction: A16.09, A16.19, A16.29.

Gel A17 02 (A17.25-A17.36):

Gel A17_04: A17.29+35 have pull-up at 185bp and A17.32+34 at 175bp and A17.29 at 235bp

Quality of reaction: A17.26 looks questionable

In preamp: A17.13-A17.15, A17.21-A17.23 all had light reactions, with A17.14, A17.15, and

A17.23 being the worst.

\# bands: 36

\# polymorphic bands: 19

\section{Primer set \#3-M55}

Bad lanes:

Gel A13 03 (A13.01-A13.42):

Gel A13_04: on full exposure there is pull-up on many at 62, 164, 315, 410 and a few pull-ups for a single sample, but they are all faint and not patterned to be an overflow from a neighboring lane.

Quality of reaction: A13.27 is blank, A13.33 is faint, A13.37 gets faint after 100bp, A13.35 and

A13.36 are present and look strong but missing a few of the more monomorphic bands.

In preamp: The following lanes had very light reactions: A13.27+A13.28, A13.35-A13.38.

Gel A15 03 (A15.01-A15.07):

Gel A15_04: On full exposure there is likely overflow on lanes A15.02 (i.e., approx. 155bp, 165, 175, 220, 265, 315, etc), A15.04 (ie. 220 and 265, etc), and A15.06 (not until after 450bp).

Quality of reaction: All look good.

In preamp: The following lanes had very light reactions: A15.06, A15.13, A15.20.

Gel A16 03 (A16.01-A16.10):

Gel A16_04: On full exposure there is much pull up, but they are not patterned to be an overflow from neighboring lanes, rather they might be a large peak in a different dye: many at 165 , and a large streak on lanes A16.05 and A16.10 at about 58-72bp.

Quality of reaction: A16.03 is missing few of the monomorphic bands even though other bands are very dark. A16.08 seems faint. When rescaled A16.09 is missing many bands.

In preamp: The following lane had a very light reaction: A16.09, A16.19, A16.29.

Gel A17 03 (A17.01-A17.12):

Gel A17_04: On full exposure there is much pull up, but they are not patterned to be an overflow from neighboring lanes, rather they might be a large peak in a different dye: few at 68 , A17.09 has a few dark bands at around 85,

Quality of reaction: A17.02 +A17.11 is missing a few of the monomorphic bands even though other bands are very dark.

In preamp: A17.01-A17.03, A17.09-A17.11 all had light reactions, with A17.02, A17.03 and A17.11 being the worst.

Note: Didn't call all missing bands for 7151.16 and 7151.17 because both were bad runs and were missing lots of bands.

\# bands: 40

\# polymorphic bands: 38 


\section{Primer set \#4 - M61}

Bad lanes:

Gel A13 01 (A13.01-A13.42):

Gel A13_04: On full exposure there is pull up on many at 62,164, 315, 410 and a few pull-ups for a single sample, but they are all faint and not patterned to be an overflow from a neighboring lane.

Quality of reaction: A13.27 is very light (almost absent) but when rescaled can see peaks, A13.33 is quite faint and A13.35 a bit faint.

In preamp: The following lanes had very light reactions: A13.27+A13.28, A13.35-A13.38.

Gel A15 01 (A15.01-A15.07):

Gel A15_04: On full exposure there is likely overflow on lanes A15.02 (i.e,. approx. 155bp, 165, 175, 220, 265, 315, etc), A15.04 (i.e., 220 and 265, etc) and A15.06 (not until after 450bp).

Quality of reaction: A15.07 very faint (almost absent) but rescaled can see some peaks - not really usable, all others look good, though A15.01+A15.03 is bit lighter but not really faint.

In preamp: The following lanes had very light reactions: A15.06, A15.13, A15.20.

Gel A16 01 (A16.01-A16.10):

Gel A16_04: On full exposure there is much pull-up, but they are not patterned to be an overflow from neighboring lanes, rather they might be a large peak in a different dye: many at 165 , and a large streak on lanes A16.05 and A16.10 at about 58-72bp.

Quality of reaction: A16.08 a bit light but might be OK, A16.06 light but OK.

In preamp: The following lane had very light reaction: A16.09, A16.19, A16.29.

Gel A17 01 (A17.01-A17.12):

Gel A17_04: On full exposure there is much pull-up, but they are not patterned to be an overflow from neighboring lanes, rather they might be a large peak in a different dye: few at 68 , A17.09 has a few dark bands at around 85,

Quality of reaction: A17.02 +A17.11 are missing a few of the monomorphic bands even though other bands are very dark.

In preamp: A17.01-A17.03, A17.09-A17.11 all had light reactions, with A17.02, A17.03, and A17.11 being the worst.

\# bands used on primer screen gels: 59

\# bands: 54

\# polymorphic bands: 46

\section{Primer set \#5 - M57}

Bad lanes:

Gel A14 03 (A14.01-A14.42):

Gel A14_04: On full exposure there is pull-up on many at 185, 225, 285, 330, 335, 390, 430, and 480 , likely overflow from neighboring lanes, but it is faint. Also a few larger pull-ups for a single sample, but they are all faint and not patterned to be an overflow from a neighboring lane.

Quality of reaction: A14.27 is blank but when rescaled can see peaks (don't use), A14.02, A14.10 are of a little lighter intensity than others, but OK to use, A14.28+A14.35+A14.36+A14.40 are missing some monomorphic bands. 
In preamp: The following lanes had very light reactions: A14.27+A14.28, A14.35-A14.38.

Gel A15 03 (A15.08-A15.14):

Gel A15_04: On full exposure there is likely overflow on lanes A15.14 (i.e., approx. 265, 315, etc), A15.04 (i.e., 220 and 265, etc).

Quality of reaction: A15.09 very faint (almost absent) but rescaled can see some peaks - not really usable, all others look good.

In preamp: The following lanes had very light reactions: A15.06, A15.13, A15.20.

Gel A16 03 (A16.11-A16.20):

Gel A16_04: On full exposure there is little pull up, and it tends to be scattered

Quality of reaction: At around $180 \mathrm{bp}$, lane A16.19 fades out, but top looks great.

In preamp: the following lane had very light reaction: A16.09, A16.19, A16.29.

Gel A17 03 (A17.13-A17.24):

Gel A17_04: On full exposure there is much pull up, but they are not patterned to be an overflow from neighboring lanes, rather they might be a large peak in a different dye: large patch between 50-60bp for A17.21-A17.23, and A17.14 has a few at 90 and 125-135bp.

Quality of reaction: A17.14 +A17.23 are missing a few of the monomorphic bands even though other bands are v. dark.

In preamp: A17.13-A17.15, A17.21-A17.23 all had light reactions, with A17.14, A17.15, and A17.23 being the worst.

\# bands used on primer screen gels: 42

\# bands: 44

\# polymorphic bands: 44

\section{Primer set \#6 - M60}

Bad lanes:

Gel A14 02 (A14.01-A14.42):

Gel A14_04: On full exposure there is pull-up on many at $185,225,285,330,335,390,430$, and 480 , likely overflow from neighboring lanes, but it is faint. Also a few larger pull-ups for a single sample, but they are all faint and not patterned to be an overflow from a neighboring lane.

Quality of reaction: A14.27 is blank but when rescaled can see peaks (don't use), A14.36 (might not use)+A14.40 are missing many bands, A14.28+A14.35 are missing some monomorphic bands.

In preamp: the following lanes had very light reactions: A14.27+A14.28, A14.35-A14.38.

Gel A15 02 (A15.08-A15.14):

Gel A15_04: On full exposure there is likely overflow on lanes A15.14 (i.e,. approx. 265, 315, etc) , $\bar{A} 15.04$ (i.e,. 220 and 265, etc).

Quality of reaction: A15.13 has high background, all others look good.

In preamp: The following lanes had very light reactions: A15.06, A15.13, A15.20.

Gel A16 02 (A16.11-A16.20):

Gel A16_04: On full exposure there is little pull-up, and it tends to be scattered Quality of reaction: All look OK, but A16.19 is missing few bands, might be OK In preamp: The following lane had very light reaction: A16.09, A16.19, A16.29. Gel A17 02 (A17.13-A17.24): 
Gel A17_04: On full exposure there is much pull up, but they are not patterned to be an overflow from neighboring lanes, rather they might be a large peak in a different dye: large patch between 50-60bp for A17.21-A17.23, and A17.14 has a few at 90 and 125-135bp.

Quality of reaction: A17.23 is missing a few of the monomorphic bands even though other bands are very dark, probably OK, A17.15 missing lots of bands, might not want to use

In preamp: A17.13-A17.15, A17.21-A17.23 all had light reactions, with A17.14, A17.15, and A17.23 being the worst.

\# bands used on primer screen gels: 23

\# bands: 28

\# polymorphic bands: 28

\section{Primer set \#7 - M62}

Bad lanes:

Gel A14 01 (A14.01-A14.42):

Gel A14_04: On full exposure there is pull-up on many at 185, 225, 285, 330, 335, 390, 430, and 480 , likely overflow from neighboring lanes, but it is faint. Also a few larger pull-ups for a single sample, but they are all faint and not patterned to be a overflow from neighboring lane.

Quality of reaction: A14.27 is blank but when rescaled can see peaks (don't use)

In preamp: The following lanes had very light reactions: A14.27+A14.28, A14.35-A14.38.

Gel A15 01 (A15.08-A15.14):

Gel A15_04: On full exposure there is likely overflow on lanes A15.14 (i.e. approx. 265, 315, etc), A15.04 (i.e. 220 and 265, etc).

Quality of reaction: all good.

In preamp: The following lanes had very light reactions: A15.06, A15.13, A15.20.

Gel A16 01 (A16.11-A16.20):

Gel A16_04: On full exposure there is little pull up, and it tends to be scattered Quality of reaction: All look OK

In preamp: The following lane had very light reaction: A16.09, A16.19, A16.29.

Gel A17 01 (A17.13-A17.24):

Gel A17_04: On full exposure there is much pull up, but they are not patterned to be an overflow from neighboring lanes, rather they might be a large peak in a different dye: large patch between 50-60bp for A17.21-A17.23, and A17.14 has a few at 90 and 125-135bp.

Quality of reaction: OK

In preamp: A17.13-A17.15, A17.21-A17.23 all had light reactions, with A17.14, A17.15, and

A17.23 being the worst.

\# bands used on primer screen gels: 19

\# bands: 19

\# polymorphic bands: 19 


\section{STEP \#2 - Removal of poor runs for analysis}

\section{August 2003 - Using Comparison “2003working”}

1. Started by excluding primer set M59 because it was poor across all samples, with many ambiguous bands

2. Remove junk repeats -

6933 - across all primer sets, 6933.17 is not usable, decided to exclude from our repeat list, and removed from comparison window

6934 - across all primer sets, 6934.17 is not usable, decided to exclude from our repeat list, and removed from comparison window

6935 - across all primer sets, 6935.17 is not usable, decided to exclude from our repeat list, and removed from comparison window

7151 - across all primer sets, 7151.16 and 7151.17 are not usable, decided to exclude from our repeat list, and removed from comparison window, also 6941.17 (run 2) which is a second extraction of 7151 had a poor run and excluded it as well

7163 - kept all 4 repeats for now, but 7163.15 is missing run for M61

7153 and 9938 are 2 extractions of Hiapo. 7153 had poor preamp and missing lots of bands, while 9938 good preamp, decided to remove 7153 from comparison

7160 ran $2 x$ with same DNA extraction. Run 3 of 3 was poor so it was removed leaving 2 runs. $7159-2$ runs with same DNA, neither good, removed both from analysis.

7164 and 7165 - Removed from comparison because so many missing bands indicate bad run.

Once poor runs were removed, we examined each primer set looking for missing band discrepancies.

\section{STEP \#3 - Final decision of confusing bands - to keep or not use}

\section{Primer set M3}

Band

63.8

75.8

75.8

75.8

84.4

118.5

118.5

138.2

144.2

\section{Lane}

A16.21 v 22 21absent, 22present

A15.21 v other Makoa, 15.21 absent but big nearby peak, masking?

A17.30 called present, shoulder but clear band in repeats

A10.18 no repeats, but shoulder, so called present

A15.19 called present, shoulder, but clear band in repeats A 16.25 v 17.31 clearly missing in 16.25 , and present in 17.31

A15.21

A 15.20

A $16.21 \mathrm{v} 22$ clearly absent in both

A10.13 present $\mathrm{v} 17.30$ absent but possibly masked by big shoulder following

A16.21 v 2221 present, 22 absent 


\begin{tabular}{|c|c|c|}
\hline \multicolumn{3}{|c|}{ Primer set M5 } \\
\hline Band & Lane & Decision \\
\hline 59.3 & misc. & no discrepancies between repeats, clear in others \\
\hline 88.8 & A10.02 & called present because large masking peak immediately before it, \\
\hline 94.1 & A16.21v22 & shoulder in 22 , called present \\
\hline 101.8 & A17.36 & absent, could be shoulder, called band present \\
\hline 106.9 & A17.31 & a bit different in size but present due to repeats \\
\hline 106.9 & A 15.21 & absent even though present in other repeats \\
\hline 114.5 & A17.36 & shoulder but called present \\
\hline 116.9 & A16.21v22 & absent in 22 , undecided \\
\hline 118.5 & $\mathrm{~A} 16.21+22$ & only ones missing, called absent \\
\hline 125.9 & A15.21 & shoulder called present based on repeats \\
\hline 128.2 & misc. & don't use class \\
\hline 130.1 & A10.32 & shoulder, called present because of repeats \\
\hline 130.1 & A16.21 & shoulder, called present because of repeat \\
\hline 147.6 & A17.31 & missing (but called present) versus 16.35 present \\
\hline 166.5 & $\mathrm{~A} 16.21+22$ & very small but called present \\
\hline 166.5 & A17.29 & very small but called present based on repeat \\
\hline 185.2 & misc. & don't use class \\
\hline 189.0 & A10.26 & very small but called present due to repeats \\
\hline 205.2 & A16.21v22 & very small in 21 , called present \\
\hline 230.1 & A16.21v22 & absent in 22 , undecided \\
\hline 249.4 & A16.21v22 & absent in both \\
\hline
\end{tabular}

\section{Primer set M55}

Band

72.5

84.41

99.77

110.67

115.0

121.6

129.8

131.41

131.40

187.18
Lane

A17.04

A13.37

A13.32

A16.02

A13.37

A13.40

A16.07

A 13.41

A16.01

A13.40

A16.01

Primer set M57

\section{Band}

56.2

58.1

79.9

89.65

\section{Lane}

misc.

misc.

A14.22

A16.01

\section{Decision}

present but small, called as present

repeat with presence elsewhere, called this small peak present repeat with presence elsewhere, called this small peak present repeat with presence elsewhere, called this small peak present repeat with presence elsewhere, called this small peak present no peak present, but questionable run with no repeat repeat with presence elsewhere, called this small peak present peak present, but size shift, called present repeat with presence elsewhere, called this small peak present no peak present, but questionable run with no repeat repeat with presence elsewhere, bad run, did not call peak, probably just use A16.02 b/c better run for this primer set also decided not to use the following band classes due to shoulders $77.9,91.5,105.9,125$

\section{Decision}

shoulder, might use for pedigree, remove band class now using for now

only one not present, called absent

one of Lu ukia runs, called present because definite in other run 
92.37 misc. part doublet below, can't call and be sure, don't use

118.66

144.9

194.5

225.7
A17.24

A14.40

A14.40

A14.33

\section{Primer set M60}

Band

61.7

90.5

100.7

103

104.4

105

113

185.5

306

307

313
Lane

A14.33

A16.15 v A17.19 faint but present, call present

A14.22 strong run, but no peak present, call absent

A14.39

misc

misc.

misc.

A 14.40

A14.37

small, called present

called present even if small, different from 102.7

shoulder, took out

kept, might be problem though

small, called present

likely leakover, don't use

A16.11vA16.12 likely leakover, don't use

A16.18v14.03v15.12 likely leakover, don't use

misc.

misc.

A14.40

good polymorphism, consistent across repeats

good polymorphism, consistent across repeats

definitely not there

also decided not to use the following band classes due to shoulders $61.7,91.7,159.5$

for pedigree comparisons, might want to check these:

185.5

\section{Primer set M61}

Band

57.3

57.3

61.8

65.4

77.1

81.4

84.6

85.7

86.4

99.3

100.8

102.6

105.9
Lane

A13.40

A16.05vA17.07 not present in A16.05 left uncalled, but present A17.07

present in A13.09+13.26, not in 15.06, left uncalled

A16.01v02 not in 02, left uncalled

misc.

misc.

A13.16

misc.

misc.

misc.

misc.

A13.40

misc.

A13.33 shoulder but recalled and used for all shoulder but recalled and used for all only lane with good polymorphism small, but using, call present shoulder, but recalled and used for all shoulder, but recalled and used for all shoulder, but recalled and used for all, except A13.40 small but called present shoulder, but recalled and used for all very small but called present 


$\begin{array}{lll}111.8 & \text { A13.40 } & \text { small but called present } \\ 114.7 & \text { A15.05 has but } 13.03+16.08 \text { don't use } \\ 127.7 & \text { A13.32 has but } 13.13+17.06 \text { don't use } \\ 139.3 & \text { A13.40 } & \text { small but called present } \\ 145.4 & \text { A16.01v.02 } & \text { small in .02 but called present } \\ 145.4 & \text { A13.33 } & \text { small but called present } \\ 145.4 & \text { A13.40 } & \text { small but called present } \\ 153.1 & \text { A16.01v.02 } & \text { present in .02, shoulder in .01 called present in both } \\ 153.1 & \text { A16.04 } & \text { small and shoulder but called present } \\ 157.5 & \text { A16.01v02 } & \text { not in 02, left uncalled } \\ 158.8 & \text { A16.04 } & \text { small, but called present } \\ 183.1 & \text { A13.28 } & \text { can be small, but is absent in this lane, didn't call } \\ & \text { A16.05 } \mathrm{v} \text { A17.07 present in 17.07, but not in 16.05, left } 16.05 \text { not called } \\ & \text { also decided not to use the following band classes due to shoulders } \\ & \text { 114.7+127.7+200.4+315.0 (not shoulder but mixed presence in repeats) } \\ & \text { for pedigree comparisons, might want to check these: }\end{array}$

Primer set M62

\begin{tabular}{|c|c|c|}
\hline Band & Lane & Decision \\
\hline 61.4 & A14.40 & clearly missing, didn't call \\
\hline 69.0 & A16.14 & clearly present, kept band \\
\hline 88.6 & misc. & removed, not consistent, very small \\
\hline 107.5 & \multicolumn{2}{|c|}{ A16.15 no band v A17.19 big band ???? } \\
\hline 107.5 & A16.11 & called small band in 12 present \\
\hline 124.3 & \multirow{2}{*}{\multicolumn{2}{|c|}{$\begin{array}{l}\text { mult samples missing including Kauila repeats, keep as absent polymorphism } \\
\text { A14.40 clearly present, kept called } \\
\text { gel A15\# band very small, but almost all samples repeats, so called present even } \\
\text { though extremely small }\end{array}$}} \\
\hline 146.2 & & \\
\hline 313.0 & A 14.40 & not present \\
\hline 323 & $\begin{array}{l}\text { A14.34 } \\
\text { also dec } \\
88.6 \text { (ve }\end{array}$ & $\begin{array}{l}\text { er repeats, is vsmall called present } \\
\text { ot to use the following band classes due to shoulders: } \\
\text { all) }\end{array}$ \\
\hline
\end{tabular}

\section{STEP \#4 - Consensus patterns for individuals with repeats}

(Reduced total \# in comparison from 58 to 41 individuals)

Kauila - kept 6941, removed 7151 samples

7151.13 - M57(1)

7151.15 - M3(1) M61(1)

6941 - none, kept

Keawe - all 3 identical except 6934.15

6934.13 - keep

6934.15 - M3 missing all 


$$
\begin{aligned}
& \text { 6934.16 - none } \\
& \text { Kēhau - kept } 6945 \\
& 6945 \text { - keep } \\
& 7157 \text { - none } \\
& 9940 \text { - M3(1) M57(1) M60(1) }
\end{aligned}
$$

Kolohe - all 3 identical, kept 6933.13 (removed 6933.15 and 6933.16)

Lōkāhi - kept 6944

$6944-\mathrm{kept}$

$7171-$ M57(1)

Makoa - kept 7163.13

$7163.13 \mathrm{kept}$

7163.15 - M3(2) M5(1) M60(1) (missing M61)

7163.16 - none

7163.17 - M57(1) M60(1)

Wa'alani - all 3 same, kept 6935.13 (removed 6935.15 and 6935.16)

Ao - called present the M5 and M60 band in 9941 and then removed 7168

9941 - M5(147bp) M60(103bp)

7168 - M3(1) M61(2) M62(1)

Lu ukia - called present 3 missing bands in run 1 and then remove run 2

Run 1 - M3(63.8) M55(187.1) M57(89)

Run 2 - M3(1) M5(1) M60(1) M61(2)

'Oli - use 9939 after calling the M60 band present

9939 - M60(105)

$6942-$ M57(1)

\section{AFLP TIMELINE DETAILS}

September-October 2001 -New digestion ligation of 4 individuals (4086 `Ele`ele, 4110 Kauila, 4116 Noe, 4131 Hiapo) repeated 3 times, using PCl extracted samples. Screened with 6 Eco/Taq primer sets (A,B,C,D,E,F). Ran on ABI gels A1\# and A2\#.

7-8 January 2002 - First run through of 31 samples, starting from new digestion/ligation for each (Eco/Mse). 25 samples were from Nov 2001 Qiagen column extraction and 7 were from $\mathrm{PCl}$ extractions (4 being repeats from above). A1t-A4\# - Eco/Taq digestion/ligation not proceeded with.

A1m-A1\# - Eco/Mse was taken through to ABI gels, with 6 primer sets: M5, M9, M3, M6, M7, M1. Ran on ABI gels A3\# and A4\#.

A1m-A2\# - repeats were with $4 \mathrm{PCl}$ samples and not taken past digestion/ligation.

A1m-A3\# - repeats were with $4 \mathrm{PCl}$ samples and not taken past digestion/ligation.

27 January 2002 -Run 16 new samples, all are PCl extracted samples.

A1t-A5\# - Not taken past digestion/ligation.

A1m-A4\# - Not taken past pre-amplification because of poor smear.

4-7 February 2002 -Run same $16 \mathrm{PCl}$ samples. Again discarded and decided to re-extract the samples from blood using Qiagen for similarity to other extracted samples.

A1t-A6\# - Not taken past digestion/ligation. 
A1m-A5\# - Not taken past pre-amplification because of poor smear. Did retry preamplification with differing volumes of digestion/ligation to see if amount of $\mathrm{d} / 1$ affected preamp, not successful.

12 February 2002 -Ran second set of Qiagen extracted and associated repeats. A1t-A7\# and A1tA8\# - Not taken past digestion/ligation.A1m-A6\# and A1m-A7\# - Eco/Mse was taken through to ABI gels, with 6 primer sets: M5, M9, M3, M6, M7, M1. Ran on ABI gels A5\# and $\mathrm{A} 6 \#$

4 April 2002 -Wanted to test effect of primer concentration at pre-amplification stage and looked at repeatability between 2 repeats of each individual. Tested range of final concentrations from 0.2 to $5 \mathrm{uM}$. Run on ABI gel A7\#. Decided to go with $1 \mu \mathrm{M}$ concentration (i.e. $1 \mu \mathrm{l}$ of $20 \mu \mathrm{M}$ stock) as compared to $5 \mu \mathrm{M}$ for each as used prior to this.

? Month 2002 -Reran 26 (+3 repeats) and did pre-amplification with them, but not any further.

6 September 2002 -Tested a 4 individuals using both HPLC and non-HPLC purified pre-A primers to see if necessary. Run on ABI gel A8\#. No major difference seen to justify more expensive purification.

31 October $2002-$ Reran all 45 samples +4 new samples +5 repeats. A1m-A12\# - Some didn't pre-amp very well so did varied amounts of d/1 template going into pre-amp. Did 3 primers: M5, M6, M9. Ran on ABI gels A10\# (sent over A9\#-A11\# to test dilution for gel loading). On 25 April 2003, did 6 new selective amp primers. A1m-A13\# - Didn't go past pre-amplification, until 22 April 2003.

7 November 2002 -Run 8 more samples with 5 repeats. Not taken into pre-amplification, only digestion/ligation done, until 22 April 2003.

4 March 2003 - Tested 36 new (-C/-A or -C/-C) primer on samples 6943 Keawe, 6935 Wa alani, 6942 'Oli, 6946 Pōmaika i, and 6950 Laha. Ran on ABI gel A12\#. Picked out 6 to work with: NED- M55 and M57, FAM- M59 and M60, HEX- M61 and M62. All can be run with a pre-amplification of $-\mathrm{C} /-\mathrm{C}$.

31 March 2003 - (A1m-A15\#) Run 4 old samples that have had problem with preA and 4 new extractions of old samples that have been very different from other samples in past, possibly due to poor extractions (low concentration).

14 April 2003 - (A2m1-A15\# and A2m2-A15\#). Pre-amplification was unsuccessful. Repeated with same set up the next day.

15 April 2003 - (A2m1-A15b\# and A2m2-A15b\#). Pre-amplification was unsuccessful. Repeated one more time on 21 April 2003.

16 April 2003 - (A1m-A16\#) Repeat of A15\# samples. Possibly contaminated some of the samples during ligation set up. Will carry through samples to use to test pre-amplification and then discard these.

21 April 2003 -Even with all new reagents, pre-amplification was still unsuccessful. In this reaction, also ran the A1m-A16\# samples to see if might be bad $\mathrm{D} / \mathrm{L}$ rather than preA. Because A16\# worked and not A15\#, likely the problem was with the digestion/ligation. So need to start these samples over (can't use A16\# because possible contamination).

22 April 2003 -Repeat of A15\# samples.

22 April 2003 -Preamp of good reactions from past to prepare for 6 new selective amps.

24 April 2003 -Pre-amplification still didn't work. Redo digestion/ligation with all new reagents.

25 April 2003 -6 new primer sets run on A12\#. Run on ABI gels A13\# and A14\#.

28 April-1 May 2003 -9 primer sets run on A13\#. Run on ABI gel A15\#.

29 April-1 May 2003 -\#). 9 primer sets run on A14\#. Run on ABI gel A16\#. 
29 April 2003 -Repeat of A15\# samples with new reagents.

30 April 2003 -Pre-amp finally worked.

30 April-1 May 2003 -9 primer sets run on A18\#. Run on ABI gel. 